\title{
High Electron Gain from Single Walled Carbon Nanotubes Stimulated by Interaction with an Electron Beam
}

\author{
by \\ Mario Michan \\ B.A.Sc. (Engineering Physics), The University of British Columbia, 2006
}

A THESIS SUBMITTED IN PARTIAL FULFILLMENT OF THE

REQUIREMENTS FOR THE DEGREE OF

MASTER OF APPLIED SCIENCE

in

The Faculty of Graduate Studies

(Electrical and Computer Engineering)

THE UNIVERSITY OF BRITISH COLUMBIA

(Vancouver)

April 2010

(C) Mario Michan, 2010 


\begin{abstract}
Carbon nanotubes, hollow cylindrical structures made of carbon atoms with diameters in the order of a few nanometres, are attractive candidates for electron emission applications. A detailed characterization of the phenomenon so-called electron-stimulated field-emission from carbon nanotubes is presented in this thesis. An electron beam hitting the tip of a nanotube biased near the threshold of field-emission can stimulate the emission of a large number of electrons from the nanotube tip. The result of this interaction is that high electron gains can be obtained with very small stimulating electron beam currents. Electron gains of up to 2300 were recorded. This is important as this effect could form the basis of several vacuum nanoelectronic devices. Possible direct and indirect electron-nanotube interaction mechanisms responsible for this high gain are discussed and substantiated through simulations. The design and assembly of an ultra high vacuum apparatus for more controlled future experiments is also presented.
\end{abstract}




\section{Table of Contents}

Abstract .................................................................................................................................................. ii

Table of Contents....................................................................................................................................iii

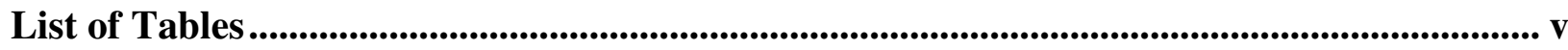

List of Figures .................................................................................................................................................... vi

Acknowledgements ....................................................................................................................................... $\mathrm{x}$

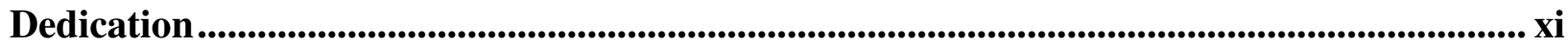

Statement of Co-Authorship ...................................................................................................................... xii

1 Introduction ........................................................................................................................................... 1

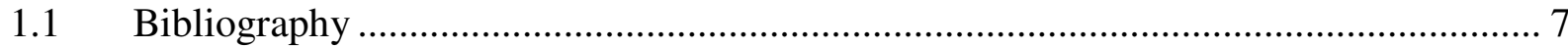

2 High Electron Gain from Single Walled Carbon Nanotubes Stimulated by Interaction with an Electron Beam ..................................................................................................................... 8

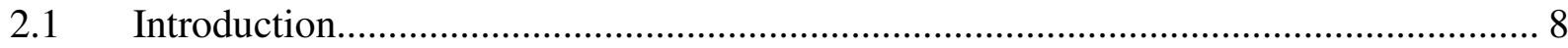

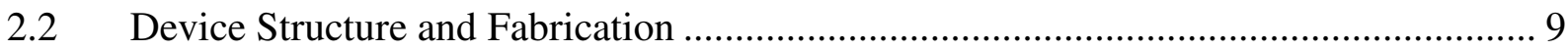

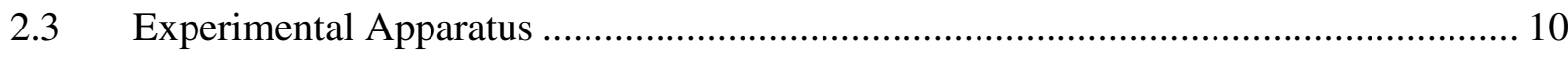

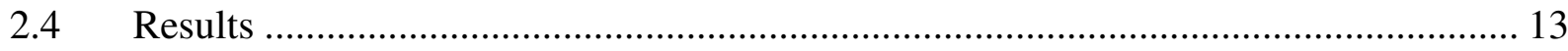

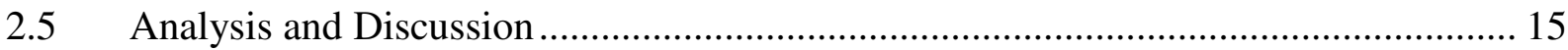

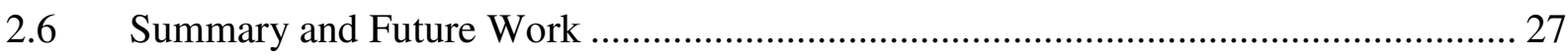

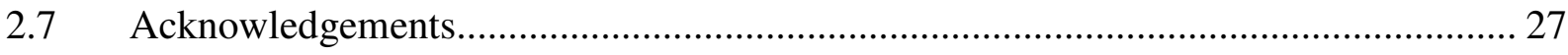

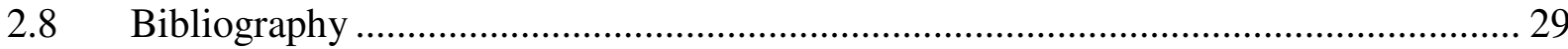

3 Conclusion and Ongoing/Future Work ............................................................................ 31

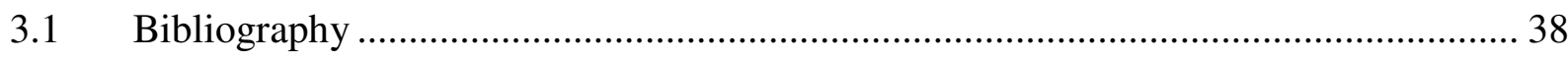

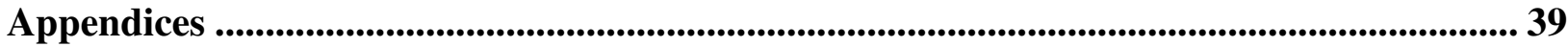

A NanoLab: Ultra-High Vacuum Apparatus for Nanostructure Research........................ 39

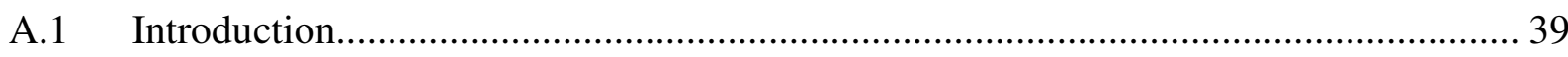

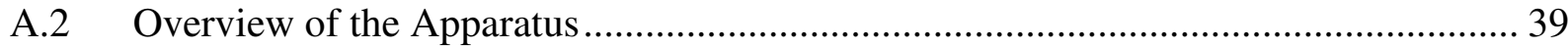

A.2.1 UHV System and Supporting Frame ……………............................................ 40

A.2.2 Sample Transfer and Manipulation …………………………………………... 46

A.2.3 Probes and Detectors ………………………………....................................... 47 
A.2.4 Auxiliary Equipment ........................................................................ 48

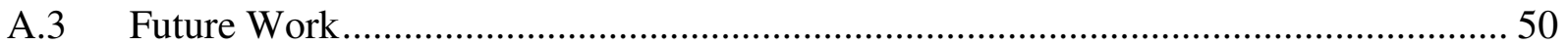

B Supplementary Material for Chapter 2 ................................................................................51

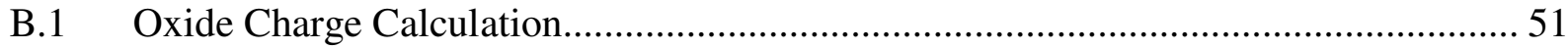

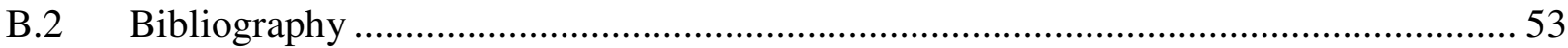




\section{List of Tables}

Table 1.1: Properties of commonly used electron sources ................................................. 3 


\section{List of Figures}

Figure 1.1: Mechanisms of electron extraction from a metal surface. a) The electron is supplied with the work function energy to overcome the emission barrier. This energy can be supplied by increasing the temperature of the source, thermionic emission, or by irradiating the source with photons matching the energy gap, photo-emission. b) The emission barrier is decreased by applying a bias across the vacuum reducing the effective work function. This type of emission mechanism is called Schottky emission and the electron is still supplied with enough energy to overcome the barrier. c) When sufficient bias is applied the electron can tunnel through the barrier. This mechanism is called field emission and sources that rely on it are called cold field emitter as they can operate at room temperature.

Figure 2.1: a) Cross-sectional schematic view of the device structure. b) SEM image of a device (top view). The molybdenum cathode is visible on the left with a catalyst island and several nanotubes a few micrometers long each attached to it and lying on the $\mathrm{SiO}_{2}$ substrate. The anode is on the right side (not in the field of view here) at approximately $30 \mu \mathrm{m}$ from the nanotubes.

Figure 2.2: Schematic of the experimental apparatus with the device under test. The diagram shows the Keithley 6517A electrometer, which was used as voltage source and current meter, the device with CNTs and integrated electrodes, and the Philips 525 SEM with the high energy electron beam and the secondary electron detector. Additionally the current guard circuit and common ground are shown.

Figure 2.3: SEM image of an SFE event obtained from the device shown in Figure 2.1 (The image is rotated by $\sim 45 \mathrm{deg}$ compared to Figure 2.1). SFE started when the electron beam hit the tip of the nanotube and continued for about $2 \mathrm{msec}$. The bright stripe in the image is caused by emitted electrons saturating the secondary electron detector as the primary beam scans beyond the nanotube tip for that time. This SFE event happened with a primary beam energy of $5 \mathrm{keV}$, 
spot size of $5 \mathrm{~nm}$ and primary beam current of $0.8 \mathrm{pA}$. The maximum emission current measured in this experiment was $12.2 \mathrm{pA}$.

Figure 2.4: Cathode current during an SFE experiment with a 5-keV / 5-nm-spot primary beam and $5 \mathrm{~nm}$ spot size scanning at $0.032 \mathrm{sec} /$ line and 250 lines per frame. The SFE current peaks occur every $8 \mathrm{sec}$, which correlates with the primary beam scanning over the tip of the nanotube.

Figure 2.5: Measured maximum electron gain for a single SFE event and average gain for SFE at different primary beam energies. The electron gain is calculated as the ratio of the number of electrons passing through the cathode in the circuit of Figure 2.2 per the number of primary beam electrons reaching the sample during one interaction cycle. 15

Figure 2.6: a) Zoomed-in image of the SFE spot of Figure 2.3 with the primary beam scanning direction and emitting nanotube position as indicated. b) Pixel intensity map extracted from image. c) Average intensity of each row as a function of distance from the top edge of the SEM image shown in (a). 16

Figure 2.7: a) Electron trajectories calculated with a Monte Carlo simulation of the primary beam interaction with the $\mathrm{SiO}_{2}$ substrate using the program CASINO [16]. The primary beam energy is $5 \mathrm{keV}$ and the spot size 5nm; 100000 electrons were used in the simulation. b) Distribution of electrons as a function of penetration depth into the substrate. The maximum depth is $\sim 520 \mathrm{~nm}$. c) Distribution of backscattered electrons (energy greater than $50 \mathrm{eV}$ ) as a function of distance from the primary beam on the substrate surface.

Figure 2.8: a) Electron trajectories calculated with a Monte Carlo simulation of 100000 electrons at $15 \mathrm{keV}$ and $5 \mathrm{~nm}$ spot size and impinging on a 2- $\mu \mathrm{m} \mathrm{SiO}_{2}$ layer on a Si substrate. b) Distribution of electrons as a function of penetration depth into the $\mathrm{SiO}_{2}$ layer. The large peak corresponds to the $\mathrm{SiO}_{2}-\mathrm{Si}$ interface.

Figure 2.9: Schematic of a model of one of the experimental devices used to simulate the electric field at the tip of the nanotube. The model closely matches the dimensions and materials used in the actual devices. 22

Figure 2.10: SEM image of the device with a floating silicon substrate (a) and top view of the calculated electric field distribution without any substrate charging: without a ground plane (b) and with a ground plane (c). As expected, the image shows a higher electric field flux (higher effective electric field) at the tip of the 
nanotube. The value of the field at the nanotube tip is comparable in both cases.

Figure 2.11: a) Schematic of the model showing the cathode electrode with a nanotube and the charge pixel directly below the tip. The charge pixel is $100 \mathrm{~nm} \times 100 \mathrm{~nm} . \mathrm{b}$ ) Charge pixel centered at the CNT tip position. c) Charge pixel $100 \mathrm{~nm}$ before the tip of the nanotube. d) Charge pixel $100 \mathrm{~nm}$ in front of the nanotube tip.

Figure 2.12: SFE with a 5-keV primary beam in line scan (32 $\mathrm{msec}$ per line) along a line passing through the nanotube tip. The nanotube tip was being hit every 32 msec by the primary beam. Every data point corresponds to the average current for a line scan. The beam was slowly moved toward the nanotube at time $\sim 100$ sec and away starting at approximately time $111 \mathrm{sec}$. It was brought back over the nanotube starting at approximately $118 \mathrm{sec}$ and away at $130 \mathrm{sec}$.

Figure 3.1: The experimental apparatus. The electron beam of the SEM is used to stimulate field-emission from the nanotubes. The emitted current is measured at the cathode with an electrometer. The nanotube forests are approximately $100 \mu \mathrm{m}$ long and cover an area of about $5 \mathrm{~mm}$ by $5 \mathrm{~mm}$.

Figure 3.2: Stimulated field-emission from nanotube forest. The maximum gain obtained was 19,000. The arrows on the figure indicate the scanning direction and position of the electron beam. The diagram is aligned with the current vs. time graph to show how the position of the beam is related to the emission current. As the electron beam scans the nanotube forest starting from the tip and advancing towards the body of the nanotubes, the emitted current decreases.

Figure A.1: Schematic diagram of NanoLab. The main components are labelled in this front-isometric view. Hoses, wires and electronic controllers are not included in the diagram. Ports that are not populated are covered with blanks not shown in the schematic.

Figure A.2: Schematic diagram of NanoLab. The main components are labelled in this back-isometric view. Hoses, wires and electronic controllers are not included in the diagram. Ports that are not populated are covered with blanks not shown in the schematic 42

Figure A.3: Large turbomolecular pump V301 and isolating gate valve. The stainless steel hose bellows connecting the turbomolecular pumps and the scroll pump are 
indicated as well as the Piranni gauge used to measure the vacuum level of the main chamber.

Figure A.4: Piranni gauge and leak valve to control pressure in the main chamber. The gate valves isolate the main chamber from the loadlock chamber, large turbomolecular pump V301 and ion pump.

Figure A.5: Loadlock, gate valve and transfer arm configuration. The gate valve isolates the loadlock for sample loading/unloading while the main chamber is held at low pressure. A small turbomolecular pump V81 (behind the loadlock) is used to evacuate the loadlock. The transfer arm transfers the sample into the main chamber where it is placed on a stage for experiments.

Figure A.6: UHV system and modular frame. The heating tape used to bake the main chamber is visible, as well as the wire-rope shock absorbers.

Figure A.7: Isometric view of the system. Electronic controllers, assembly crane and nitrogen tanks are visible. 


\section{Acknowledgements}

I wish to express my gratitude to the faculty and students of the Microsystems and Nanotechnology Group at UBC who have inspired me to continue my work in this field. I particularly thank Dr. Alireza Nojeh for his invaluable assistance, support and guidance. Deepest gratitude is also due to the members of the supervisory committee, Dr. Lukas Chrostowski and Dr. John D. Madden.

Finally, I wish to express my love and gratitude to my beloved wife; for her understanding and endless love, throughout the duration of my studies. 
Dedication

To my wife Alison 


\section{Statement of Co-Authorship}

Chapters 1, 3 and the Appendix A were written by the author. Chapter 2 is a paper submitted for publication, which is co-authored by myself, Parham Yaghoobi, Bertin Wong and Dr. Alireza Nojeh. Parham Yaghoobi and Berting Wong put together most of the experimental apparatus. Particularly, they assembled the scanning electron microscope where all the experiments for this paper were performed. Bertin Wong also performed some of the initial experiments in collaboration with me. I helped setting up the data collection system and performed all the experiments, derivations, simulations and analysis under the guidance of Dr. Alireza Nojeh. I wrote the first draft of the paper, generated the figures and participated in a collaborative effort with Dr. Nojeh that led to the final version of this paper. 


\section{Introduction}

Vacuum electronic devices have been in use since the invention of the cathode ray tube at the end of the 19th century, and great scientific advances and applications have emerged from research on this technology. Vacuum electronic devices include ubitrons, klystrons, magnetrons, microwave tubes, vacuum interrupters, radiation detectors, electron multipliers and power grid tubes $[1,2]$. Power grid tubes, more commonly known as vacuum tubes, are the workhorse of the radio frequency (RF) industry. High power and frequency transmitters and RF generators use, exclusively, vacuum tubes as these devices are considerably superior to solid state technology in terms of energy efficiency and high gain/bandwidth properties [2]. Power grid tube devices include diodes, triodes, tetrodes and pentodes. These components, combined with microwave tubes, enable high-power applications such as ground-based satellite communication systems, microwave relay stations, eavesdropping systems, and radio-signal jamming equipment. Common microwave tubes are klystrons, travelling wave tubes, magnetrons and gyrotrons [2]. These devices have not only made world-changing technologies including radar, microwave ovens and television possible, but also have remained irreplaceable. Detectors enabled by vacuum technology include gamma ray cameras, night-vision goggles, infrared detectors and scintillator-based counters. Another important category of applications includes characterization equipment such as electron microscopes. Vacuum electronic device research is an active field and applications that exclusively rely these devices are a powerful driving force for further research

As the name indicates, all vacuum electronic devices use free electrons as the charge carrier. The only other aspect that they have in common is the requirement of an electron source and a mechanism to modulate or manipulate the generated electron beam. Modulation and manipulation are done through electromagnetic fields. These fields can be generated by simple grids (as in vacuum tubes), RF cavities or electromagnetic lenses. Most of the performance increase in the last fifty years has been the result of optimizing these "electron-optical" elements. These advances in electron-optics have happened partially due to progress in computation and simulation techniques and produced impressive devices such as scanning electron microscopes 
with sub-nanometre resolution. Some of the existing limitations on the resolution of these microscopes are caused by chromatic aberrations of the electron-optical elements. Chromatic aberrations, whose appellation is due to the similarities of electron beams with photon beams in regards to their manipulation, are caused by variations of the particle energies. This spread of the particles energies is characteristic of each specific electron source type and imposes a limit on the maximum resolution achievable. Electron sources, on the other hand, have not seen as much increase in performance and most of the traditional sources used decades ago are still being used in unchanged form. Most devices use a conical cathode (electron gun) structure called Pierce geometry, which is close to optimal as it maximises the limit of current density allowed by the space charge effect $[2,3]$. The space charge effect is the result of the mutual repulsion of particles in a beam. This effect places a limit on the maximum theoretical current density for an electron beam of a given energy. Additional limitations on the current density are imposed by the ability of the particular electron source to supply electrons. Chromatic aberrations and the limit on current density are a few of the reasons why today the electron source presents the primary performance bottleneck in vacuum devices. Further progress in the field of vacuum electronics will require new electron sources and/or new modulation techniques.

Table 1.1 shows some of the most common electron sources used and their reported parameters. Figure 1.1 summarizes the associated electron emission mechanisms schematically. More detailed information can be found in books such as "Vacuum Arcs" by J.M Lafferty et al [4]. Tungsten filaments and LaB6 crystals operate based on thermionic emission and are the most common electron sources due to their low cost. The tips of these sources have a typical radius of about $5 \mu \mathrm{m}$ and they are satisfactory for many purposes $[5,6]$. A significant disadvantage of these sources is that they require very high temperatures to operate, usually in the order of $2500^{\circ}$ $\mathrm{K}$. The high temperature decreases the lifetime of all the components in vacuum device, including the electron source itself. Additionally, due to the high temperature, miniaturization of these devices is very difficult, as anodes and grids have to be maintained at low temperature to avoid re-emission of electrons and degeneration of the modulation characteristics of the device. Another main limitation is in the brightness of the beam that they can produce. Brightness is the beam current density per unit solid angle. High brightness is desirable as it directly affects the power handling capability of the device in power applications, as well as the resolution in microscopy. Higher brightness can be obtained by using a smaller tip radius and/or coating with a low-work-function material such as $\mathrm{ZrO}$ or $\mathrm{Cs}[5,7]$, in addition to applying a high electric 
field to assist in emission. The resulting sources are called Schottky emitters. These sources are usually a good compromise between performance and cost/convenience. However, they still have to operate at relatively high temperatures $(\sim 1800)$. The high temperature also leads to a high energy spread in thermionic and Schottky emitters.

\begin{tabular}{llllllll}
$\begin{array}{l}\text { Mechanism } \\
\text { of electron } \\
\text { extraction }\end{array}$ & Source type & $\begin{array}{l}\text { Brightness } \\
{\left[\mathrm{A} / \mathrm{cm}^{2} / \mathrm{sr}\right]}\end{array}$ & $\begin{array}{l}\text { Source } \\
\text { size } \\
{[\mu \mathrm{m}]}\end{array}$ & $\begin{array}{l}\text { Energy } \\
\text { spread } \\
{[\mathrm{eV}]}\end{array}$ & $\begin{array}{l}\text { Operating } \\
\text { vacuum } \\
{[\mathrm{mbar}]}\end{array}$ & $\begin{array}{l}\text { Operating } \\
\text { temperature } \\
{\left[{ }^{\circ} \mathrm{K}\right]}\end{array}$ & $\begin{array}{l}\text { Life } \\
\text { time } \\
{[\mathrm{h}]}\end{array}$ \\
$\begin{array}{l}\text { Thermionic- } \\
\text { emission }\end{array}$ & $\begin{array}{l}\text { Tungsten } \\
\text { filament }\end{array}$ & $10^{5}$ & 25 & 2.3 & $10^{-6}$ & 2700 & 100 \\
$\begin{array}{l}\text { LaB } 6 \text { tip } \\
\begin{array}{l}\text { Schottky- } \\
\text { emission }\end{array}\end{array}$ & $\begin{array}{l}\text { ZrO coated } \\
\text { tungsten tip }\end{array}$ & $10^{6}$ & 10 & 2.3 & $10^{-8}$ & 1700 & 500 \\
$\begin{array}{l}\text { Field- } \\
\text { emission }\end{array}$ & $\begin{array}{l}\text { Molybdenum } \\
\text { spindt cone }\end{array}$ & $10^{9}$ & 0.02 & 1.0 & $10^{-9}$ & 1800 & $>1000$ \\
\hline
\end{tabular}

Table 1.1: Properties of commonly used electron sources 

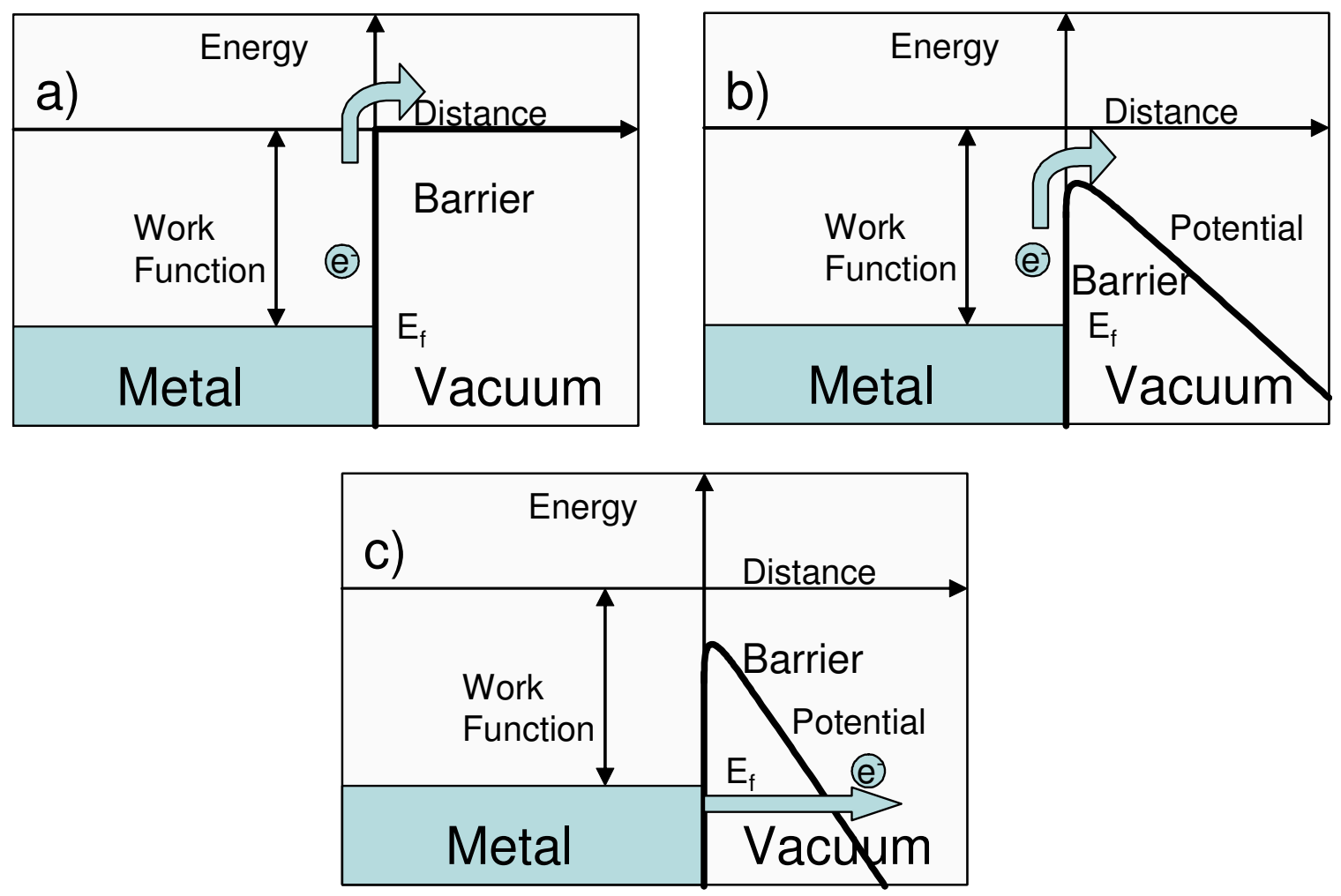

Figure 1.1: Mechanisms of electron extraction from a metal surface. a) The electron is supplied with the work function energy to overcome the emission barrier. This energy can be supplied by increasing the temperature of the source, thermionic emission, or by irradiating the source with photons matching the energy gap, photo-emission. b) The emission barrier is decreased by applying a bias across the vacuum reducing the effective work function. This type of emission mechanism is called Schottky emission and the electron is still supplied with enough energy to overcome the barrier. c) When sufficient bias is applied the electron can tunnel through the barrier. This mechanism is called field emission and sources that rely on it are called cold field emitter as they can operate at room temperature.

The highest-performance devices use cold field-electron emitters (field-emitters) as their electron source. These sources emit electrons at room temperature, which significantly increases the life span of the device. Additionally, they produce the highest brightness of all electron sources. Some common cold field-emitters are single crystal tungsten, molybdenum cones or tantalum filaments. The electrons leave the surface of these sources due to quantum-mechanical tunnelling through a thinned-down vacuum barrier because of the strong applied electric field. It is thus not necessary to provide them with the work function energy. The result is that a higher number of electrons per unit area can be extracted from the material, leading to higher brightness 
(compared to thermionic emission where only electrons in the high-energy tail of the FermiDirac distribution can be emitted), and the energy spread (variation in kinetic energy) of such electrons is very small (almost an order of magnitude less than that of thermionic emitters) due to lack of thermal broadening. This is perhaps the main reason why reliable cold field-emitters are desirable in microwave tubes; they produce a clean monochromatic electron beam [6].

A particular material that has been found to be a highly attractive candidate for electron source applications is the carbon nanotube. Carbon nanotubes are allotropes of carbon with a tubular structure. The carbon atoms are arranged in a honeycomb lattice that can be thought of as rolled graphene. Nanotubes can have a very high aspect ratio. For instance, nanotubes synthesized with length-to-diameter ratios of up to 28,000,000:1 have been reported [8]. Single-walled nanotubes (SWNTs) are formed by one sheet of graphene. Multi-walled nanotubes (MWNTs) are formed by several SWNTs in a coaxial arrangement (several concentric SWNTs form one MWNT). Nanotubes are formed entirely from sp2 hybrid bonds as in graphene. This bonding structure is stronger than the sp3 bonds found in diamond and that makes them a particularly strong and stable material. These properties combined with the high conductivity found in nanotubes make them a very good candidate for cold field-emitter sources. Due to their high aspect ratio, an externally applied electric field is enhanced hundreds to thousands of times at their tip. It is thus possible to extract electrons with low applied voltages and, since the nanotube tip radius is on the order of a few nanometers, it is possible to produce very localized electron sources. Lowvoltage operation combined with their nanoscale nature opens the door for miniaturization of vacuum electronic devices and several new applications where size is a constraint. An example application that would benefit from miniaturization is space electronics and small communication satellites.

A particular aspect of carbon nanotubes that could make them very useful not only for miniaturized vacuum electronics but also as a basic component of a new range of devices is a new modulation technique called electron stimulated field-emission (SFE). SFE was first observed by Nojeh et al. when attempting to observe field-emission spots from carbon nanotubes under a scanning electron beam. It was discovered that electron field-emission was being stimulated by the irradiation of the nanotube tip by the electron beam of an electron microscope [9]. Further experiments and simulations suggested that the field-emission was at least partially caused by a direct interaction between the electrons of the stimulating beam and the electron 
orbitals at the tip of the nanotube $[10,11]$. First-principles calculations showed that it was possible that the electrons from the beam were raising the energy levels of the nanotube, thus facilitating electron emission [11]. A very interesting aspect of this phenomenon is that it exhibits electron gain: a few electrons from the stimulating beam seem to cause a large electron emission current. This gain is important as this effect could form the basis of a nanoscale current-controlled vacuum transistor, an electron multiplier, or a highly localized electron detector. In any of these applications high gain is desirable. The SFE phenomenon has been further characterized and other types of possible interactions responsible for the electron emission have been described.

This thesis describes a detailed characterization of SFE and presents possible explanations of the experimental data obtained. The following chapter is a submitted paper where all the experiments are described. It is shown that very high electron gains (gains as high as 2,300 were measured) are possible from these devices. A systematic analysis of the interactions responsible for the high electron gain is also presented. This analysis could be used as the basis for the design of new nanoscale vacuum electronic devices.

In addition to the above-mentioned research work on nanotubes, a versatile ultra-high vacuum (UHV) system (that we refer to as the "NanoLab") has been designed and built to allow our group to perform a variety of experiments on nanostructures in a highly controlled environment (minimizing the effect of adsorbates and contaminants). An example would be further SFE experiments to shed further light on its underlying mechanisms. In this system it is possible to use several probing techniques (electron beam, photon beams, Nanomanipulation, energy analysis) simultaneously to analyse complex structures. NanoLab development and capabilities are described in Appendix A. 


\subsection{Bibliography}

[1] J. A. Eichmeier, and M. K. Thumm, (2008), Vacuum Electronics Components and Devices, Berlin: Springer.

[2] J. C. Whitaker, (1999), Power Vacuum Tubes Handbook, Philadelphia: CRC Press.

[3] J. H Moore, C. Davis, and M. Coplan, (2002), Building Scientific Apparatus, Massachutsetts: Westview Press.

[4] J. M. Lafferty, (1980), Vacuum Arcs, Theory and Application, New York: Wiley.

[5] R. Waser, (2005), Nanoelectronics and Information Technology, Weinheim:Wiley-vch.

[6] P. J. Goodw, J. Humphreys, and R. Beanland, (2001), Electron Microscopy and Analysis, London: Taylor \& Francis.

[7] S. Suzuki, C. Bower, Y. Watanabe, and O. Zhou, Applied Physics Letters, 76:4007, (2000).

[8]. X. Zheng; M. J. O'Connell, S. K. Doorn, X. Z. Liao, Y. H. Zhao, E. A. Akhadov, M. A. Hoffbauer, B. J. Roop et al. Nature Materials 3 (10), 673, (2004).

[9] A. Nojeh, W.-K. Wong, A. W. Baum, R. F. Pease, and H. Dai, Applied Physics Letters 85, $112,(2004)$.

[10] A. Nojeh, W.-K. Wong, E. Yieh, R. F. Pease, and H. Dai, Journal of Vacuum Science and Technology B 22, 3124 (2004).

[11] A. Nojeh, B. Shan, K. Cho, and R. F. W. Pease, Physical Review Letters 96, 056802 (2006). 


\section{High Electron Gain from Single Walled Carbon Nanotubes Stimulated by Interaction with an Electron Beam ${ }^{1}$}

\subsection{Introduction}

Single-walled carbon nanotubes (SWNTs) are tubular structures made of carbon atoms in a honeycomb lattice that can be thought of as rolled graphene. They have diameters of around one nanometer and can be as long as a few centimeters. SWNTs show very high strength, mechanical stability and excellent electrical conductivity. Additionally, they are excellent electron fieldemitters. Pioneering work on field-emission from nanotubes was done in the mid 1990's [1, 2, 3] and many subsequent works have further examined their interesting field-emission behaviour $[4$, 5]. Several review articles have been written on the topic, including an early review by Bonnard et al. [6] and more recent ones [7, 8]. Field-emission is a process whereby electrons are extracted from a surface under a high external electric field. The process is due to quantum tunneling and typically produces a more narrow distribution of electron energies and a more localized emission area than thermionic emission where the electrons are excited by high temperatures.

In one particular experiment, the primary beam of a scanning electron microscope (SEM) was observed to trigger field-emission from a biased SWNT lying on an insulating substrate $[9,10]$. This effect was called "stimulated field-emission (SFE)" since the bias applied to the carbon nanotube (CNT) was not strong enough to cause field-emission by itself; an additional stimulation - the SEM's primary electron beam - was required to initiate the emission process. This emission happened only when the scanning primary beam hit the nanotube tip. Therefore, it appeared on the SEM image as a bright spot - the result of a large number of emitted electrons saturating the secondary electron detector at the moment when the primary beam was on the tip of the nanotube. Stimulated field-emission has also been observed from multi-walled carbon nanotubes interacting with a SEM beam [11].

\footnotetext{
${ }^{1}$ A version of this chapter has been submitted for publication. M. Michan, P. Yahoobi, B. Wong, A. Nojeh, High Electron Gain from Single Walled Carbon Nanotubes Stimulated by Interaction with an Electron Beam
} 
As will be discussed in detail in the present report, SFE shows a high electron gain. This is interesting since such a device could form the basis of a nanoscale current-controlled vacuum transistor, an electron multiplier, or a highly localized electron detector. The stimulating mechanism was believed to be at least partially the direct interaction of the primary beam electrons with the nanotube tip. Such an interaction was investigated using first-principles calculations and it was observed that an electron entering the nanotube tip region raises the orbital energies significantly, making their occupying electrons more susceptible to tunneling out of the nanotube [12]. In this paper, we further characterize the behavior of these devices, in particular by measuring the electron gain under different conditions, and attempt to shed further light on the possible interaction mechanisms at work.

\subsection{Device Structure and Fabrication}

In order to characterize this phenomenon several experiments in multiple devices were performed. Figure 2.1 shows a schematic of a typical device used and an SEM image of such a device. The SWNT is lying on a $\mathrm{SiO}_{2}$ substrate. The substrate provides mechanical support and a mechanism for cooling down by heat exchange during emission (nanotubes on a substrate have been observed to enable the passage of higher current compared to suspended ones due to thermal relaxation through the substrate [13]). The devices have integrated cathode and anode electrodes. This arrangement, given the small distance between the electrodes, allows us to generate the necessary electric fields using a small bias voltage. In this way the operation of the SEM and the primary beam trajectory are not disturbed. The devices were fabricated using standard microfabrication processes (lithography, metal deposition and lift-off) to pattern the electrodes (molybdenum) and catalyst islands. Nanotubes were grown using chemical vapor deposition. A more detailed description of this process can be found in [14]. 


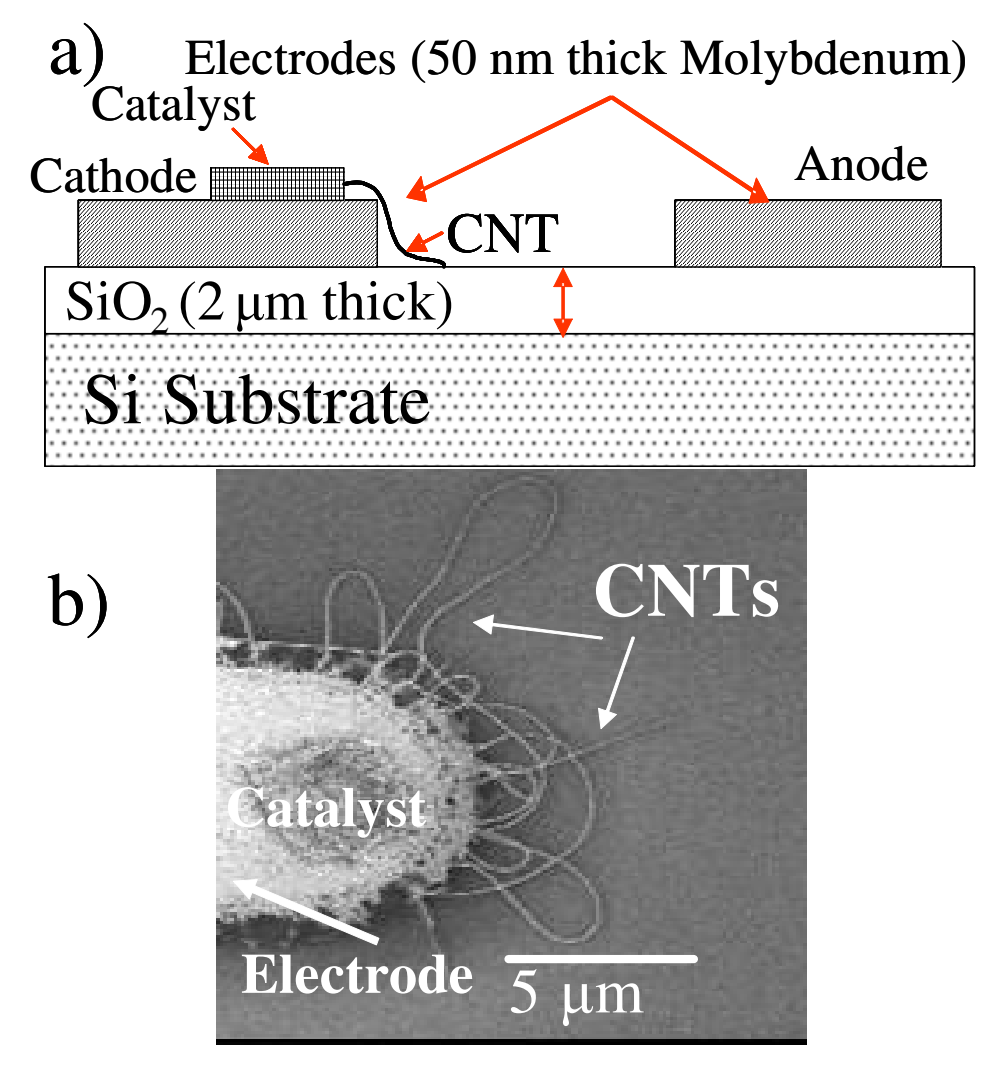

Figure 2.1: a) Cross-sectional schematic view of the device structure. b) SEM image of a device (top view). The molybdenum cathode is visible on the left with a catalyst island and several nanotubes a few micrometers long each attached to it and lying on the $\mathrm{SiO}_{2}$ substrate. The anode is on the right side (not in the field of view here) at approximately $30 \mu \mathrm{m}$ from the nanotubes

\subsection{Experimental Apparatus}

The experiments were performed in a Philips 525 SEM, which was additionally equipped with electrical feedthroughs to allow the in-situ biasing of the nanotubes and current measurements. Custom-designed sample holders were made with suitable current guards to reduce parasitics and leakage currents. A Keithley 6517A electrometer was used as voltage source and current meter. This instrument, with a $1 \times 10^{-15}$-A resolution, provided sufficient accuracy for the measurements. This electrometer was interfaced with a computer in order to record voltage and current as a function of time. Given the small magnitude of the currents measured, we had to improve the signal to noise ratio by averaging several readings, typically from 10 to 100 , and by choosing a suitable analog to digital converter integration time in the order of $20 \mathrm{msec}$. This strategy 
improves the signal to noise ratio but at the same time decreases the maximum sampling rate possible. Figure 2.2 shows the schematic of the experimental apparatus.

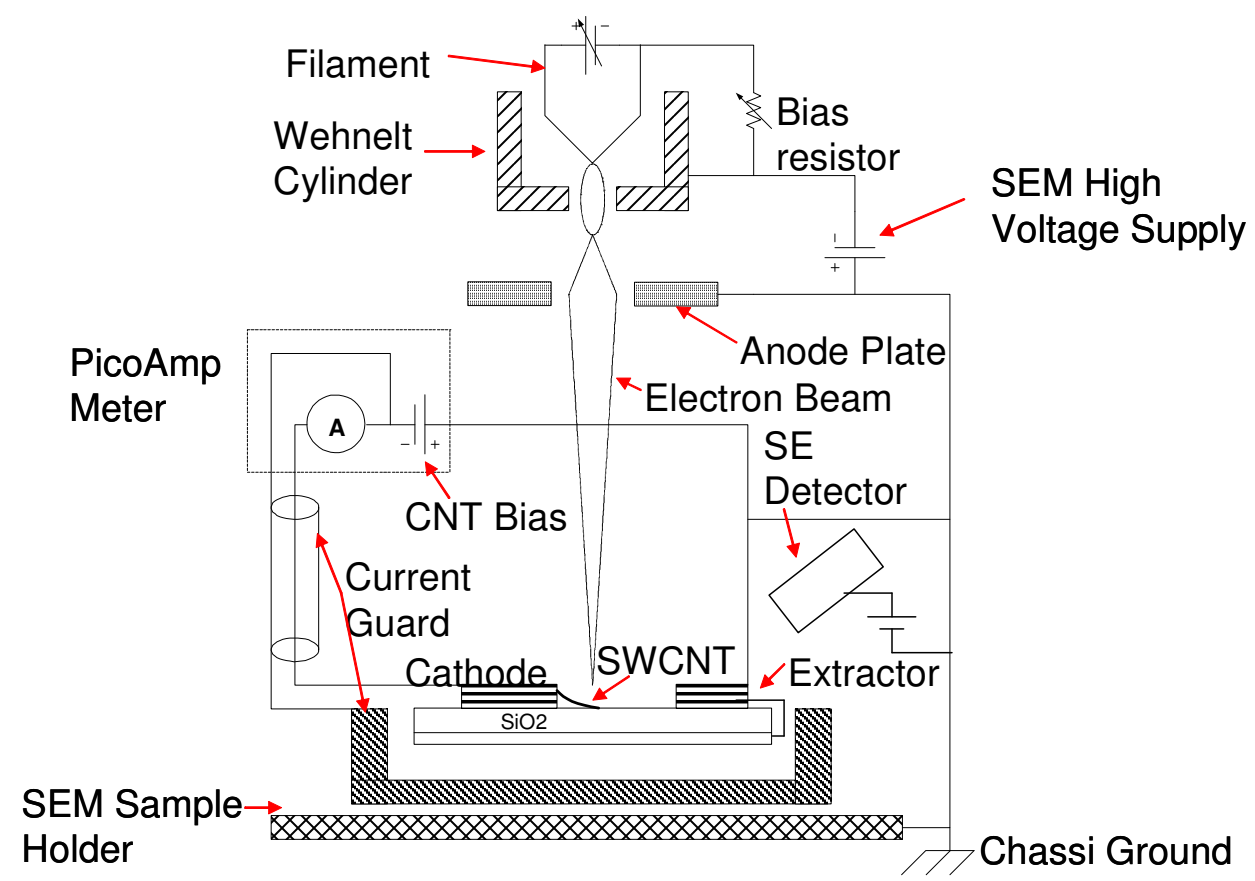

Figure 2.2: Schematic of the experimental apparatus with the device under test. The diagram shows the Keithley 6517A electrometer, which was used as voltage source and current meter, the device with CNTs and integrated electrodes, and the Philips 525 SEM with the high energy electron beam and the secondary electron detector. Additionally the current guard circuit and common ground are shown.

During a typical experiment the device under test was initially imaged with the SEM and the primary beam current was measured with a Faraday cup. The anode (extractor) was held at ground potential and the cathode (the electrode where the nanotube was attached) was biased negatively. Any emission or leakage current through the cathode was measured with the current meter. The bias was then increased slowly up to just before the threshold of regular fieldemission. At this bias, stimulated field-emission could be observed as a bright spot on the nanotube tip (Figure 2.3). (Further increasing the bias into the regular field-emission regime would, of course, lead to the SEM image being washed out since the spontaneously field-emitted electrons from the nanotube tip would flood the secondary electron detector regardless of the position of the primary beam.) As can be seen on Figure 2.3, often the emission would continue for some time after the primary beam had swept past the nanotube tip, leading to a streak on the 
image. The bright spot and line are due to secondary electron detector saturation by the portion of SFE electrons that escape the device anode and fly to the detector. The stimulated emission current was recorded as peaks that agreed with the timing of the scanning primary beam hitting the nanotube tip (Figure 2.4). This SFE event happened with a primary beam energy of $5 \mathrm{keV}$, spot size of $5 \mathrm{~nm}$ and primary beam current of $0.8 \mathrm{pA}$. The maximum emission current measured in this experiment was $12.2 \mathrm{pA}$.

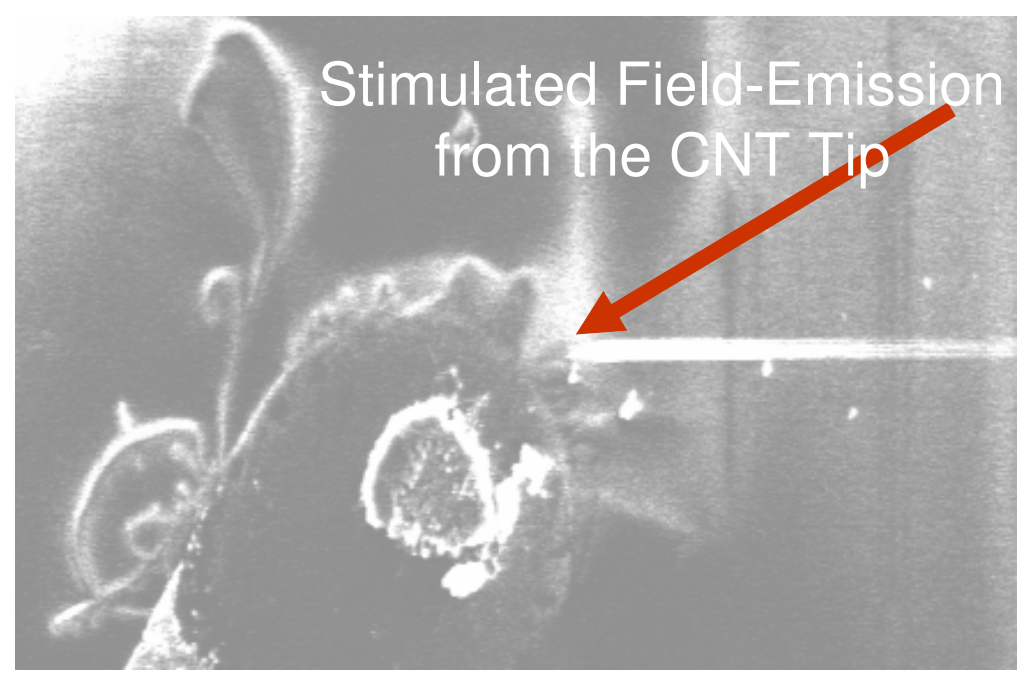

Figure 2.3: SEM image of an SFE event obtained from the device shown in Figure 2.1 (The image is rotated by $\sim 45$ deg compared to Figure 2.1). SFE started when the electron beam hit the tip of the nanotube and continued for about $2 \mathrm{msec}$. The bright stripe in the image is caused by emitted electrons saturating the secondary electron detector as the primary beam scans beyond the nanotube tip for that time. This SFE event happened with a primary beam energy of 5 $\mathrm{keV}$, spot size of $5 \mathrm{~nm}$ and primary beam current of $0.8 \mathrm{pA}$. The maximum emission current measured in this experiment was $12.2 \mathrm{pA}$. 
SFE @ 5 keV 32 msec/line 250 lines/frame

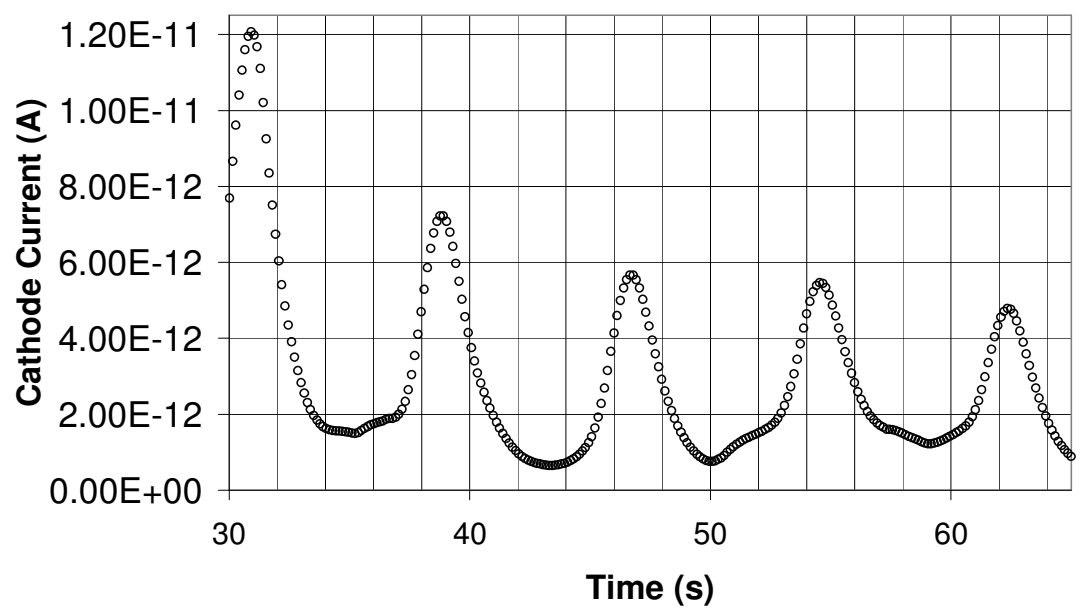

Figure 2.4: Cathode current during an SFE experiment with a 5-keV / 5-nm-spot primary beam and $5 \mathrm{~nm}$ spot size scanning at $0.032 \mathrm{sec} /$ line and 250 lines per frame. The SFE current peaks occur every $8 \mathrm{sec}$, which correlates with the primary beam scanning over the tip of the nanotube.

The experiments were performed under different conditions where several of the parameters that could potentially play a role in the emission current were varied. The scan period can be adjusted in the SEM, as well as the primary beam energy and spot size. The applied bias varied from device to device (different geometries) between -150 V and -170 V. Given the anode-cathode distance of $32.5 \mu \mathrm{m}$ this corresponds to average applied electric fields in the range of $4.6 \mathrm{~V} / \mu \mathrm{m}$ to $5.2 \mathrm{~V} / \mu \mathrm{m}$ along the nanotube, which are around the threshold of field-emission for carbon nanotubes. Obviously, due to the high aspect ratio of the nanotubes, the field at the nanotube tip is enhanced hundreds of times compared to the applied field [14, 15]. As will be further discussed, based on our simulations, this effective field at the tip of the nanotube was almost the same in all devices during SFE.

\subsection{Results}

Stimulated field-emission was clearly observed at primary beam energies of 3, 4, 5, 6 and 10 $\mathrm{keV}$. It was not observed at 1, 2 and $15 \mathrm{keV}$, indicating that either there was no SFE or it was weak and obscured by noise. Experiments performed on different devices showed some variance in SFE current amplitudes (with a few of the devices showing no field-emission at all). However, 
at any given primary beam energy most devices performed similarly and the main difference between different energies was the amplitude of the SFE current. Figure 2.4 is an example of the current measurements obtained as a function of time. For this particular experiment the primary beam energy was $5 \mathrm{keV}$ and it hit the nanotube tip every 8 seconds. This experiment produced a very clean signal. The variations in current peaks are believed to be due to instabilities (and the gradual deterioration of the nanotube tip) partially because of the poor vacuum conditions in the SEM chamber. This will be discussed further.

The electron gain was calculated as the ratio of the number of electrons passing through the cathode in the circuit of Figure 2.2 per the number of primary beam electrons reaching the sample during one interaction cycle. Figure 2.5 shows the average gain calculated from all the SFE peaks during an experiment and the maximum gain obtained in a single SFE event, both as a function of primary beam energy. The global maximum gain obtained was for a single event in an experiment at $5 \mathrm{keV}$, and was 2300. The average gain at this energy is $\sim 1000$. It can be observed that the gain increases as the primary beam energy is increased from the $3 \mathrm{keV}$ to 5 $\mathrm{keV}$ where it reaches a maximum. As the beam energy is further increased the gain decreases to a minimum average value of 40 at $10 \mathrm{keV}$. At $3 \mathrm{keV}$ and $10 \mathrm{keV}$ the maximum gain is very close to the average gain (150 and 100 for $3 \mathrm{keV}$, and 50 and 40 for $10 \mathrm{keV}$ ). The SFE at $5 \mathrm{keV}$ showed the largest difference between maximum and average gain; the SFE current peaks were very large and clear at this primary beam energy, but their amplitude decreased rapidly as the experiment progressed. The effects of line scan period and beam spot size were also characterized for the primary beam energies investigated. The scan period was varied from 2 msec to $32 \mathrm{msec}$ per line, and it did not affect the current gain. Faster scanning rates could not be investigated due to in the limitations of our experimental data acquisition setup. The primary beam spot size was varied from $5 \mathrm{~nm}$ to $50 \mathrm{~nm}$ and it also did not cause any appreciable change in the measured gain. 


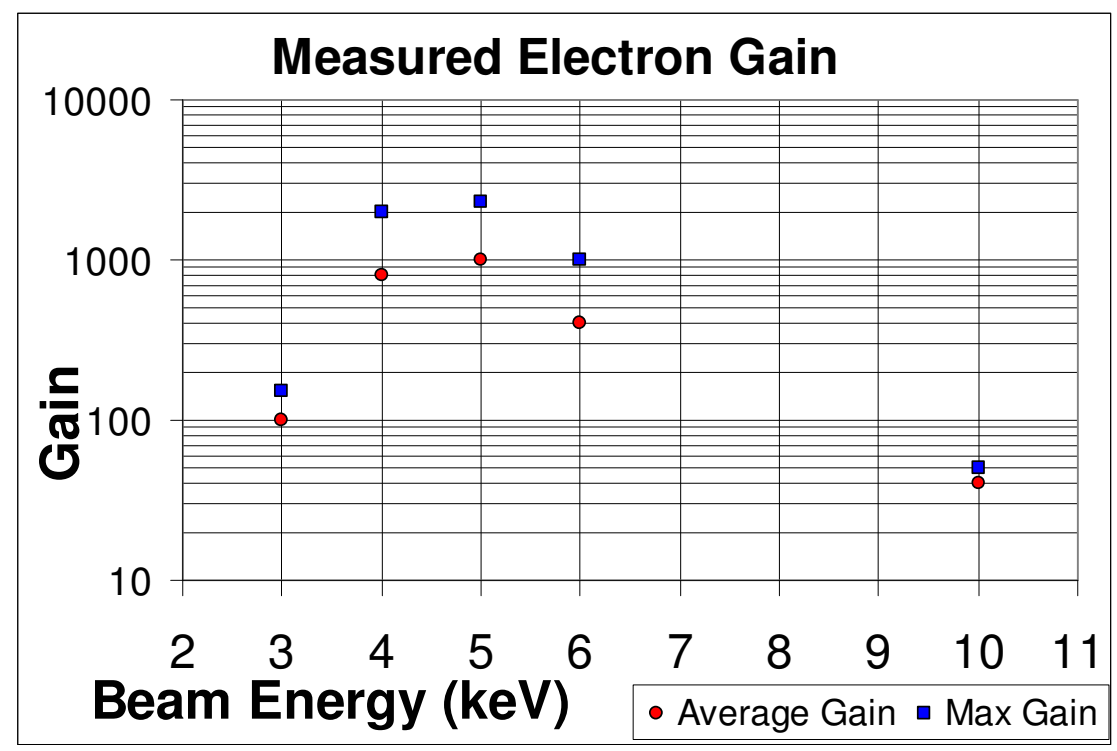

Figure 2.5: Measured maximum electron gain for a single SFE event and average gain for SFE at different primary beam energies. The electron gain is calculated as the ratio of the number of electrons passing through the cathode in the circuit of Figure 2.2 per the number of primary beam electrons reaching the sample during one interaction cycle.

The obtained gain value is based on the cathode current measured by the electrometer and is composed of the SFE current, as well as possible leakage currents between the biased electrodes through the oxide surface. Leakage currents were measured to be approximately 0.45 to $1.50 \mathrm{pA}$ : they were comparable to the primary beam current and much smaller than the SFE current. Therefore, their contribution is negligible in the gain calculation. The primary beam current was measured independently with a Faraday cup that suppresses the effect of reflected electrons. This current changes according to the adjustment of the SEM for imaging and in our various experiments it ranged from $0.45 \mathrm{pA}$ to $2.00 \mathrm{pA}$

\subsection{Analysis and Discussion}

An important factor that needs to be considered when calculating the gain is the actual interaction area of the primary beam and the nanotube. Most of the time that the primary beam is scanning the substrate, it is not interacting directly with the CNT tip. Nevertheless, the interaction area could potentially be much larger than the area of the nanotube tip due to the spreading of the beam in the substrate. 
This area can be measured using the digitally captured SEM images of SFE events: since the secondary electron detector records values at every point as the primary beam moves, and a percentage of SFE electrons are always captured by it, the SEM image provides a good measurement tool for the interaction area. Although the accuracy is limited by the pixel resolution, it is better than what the sampling rate of the electrometer allows. Figure 2.6a is a zoomed-in image of the SFE spot from Figure 2.3, and Figure 2.6b shows the pixel intensity as extracted from the image file. (As mentioned before, the horizontal streaks indicate that the emission continues for some time - in this case about $2 \mathrm{msec}$ - after the primary beam has swept past the nanotube tip; a possible explanation for this will be discussed later). The graph in Figure $2.6 \mathrm{c}$ is the average pixel intensity vs. distance from the bottom edge of the image (the value at each point is the average intensity of all the pixels in the corresponding row).

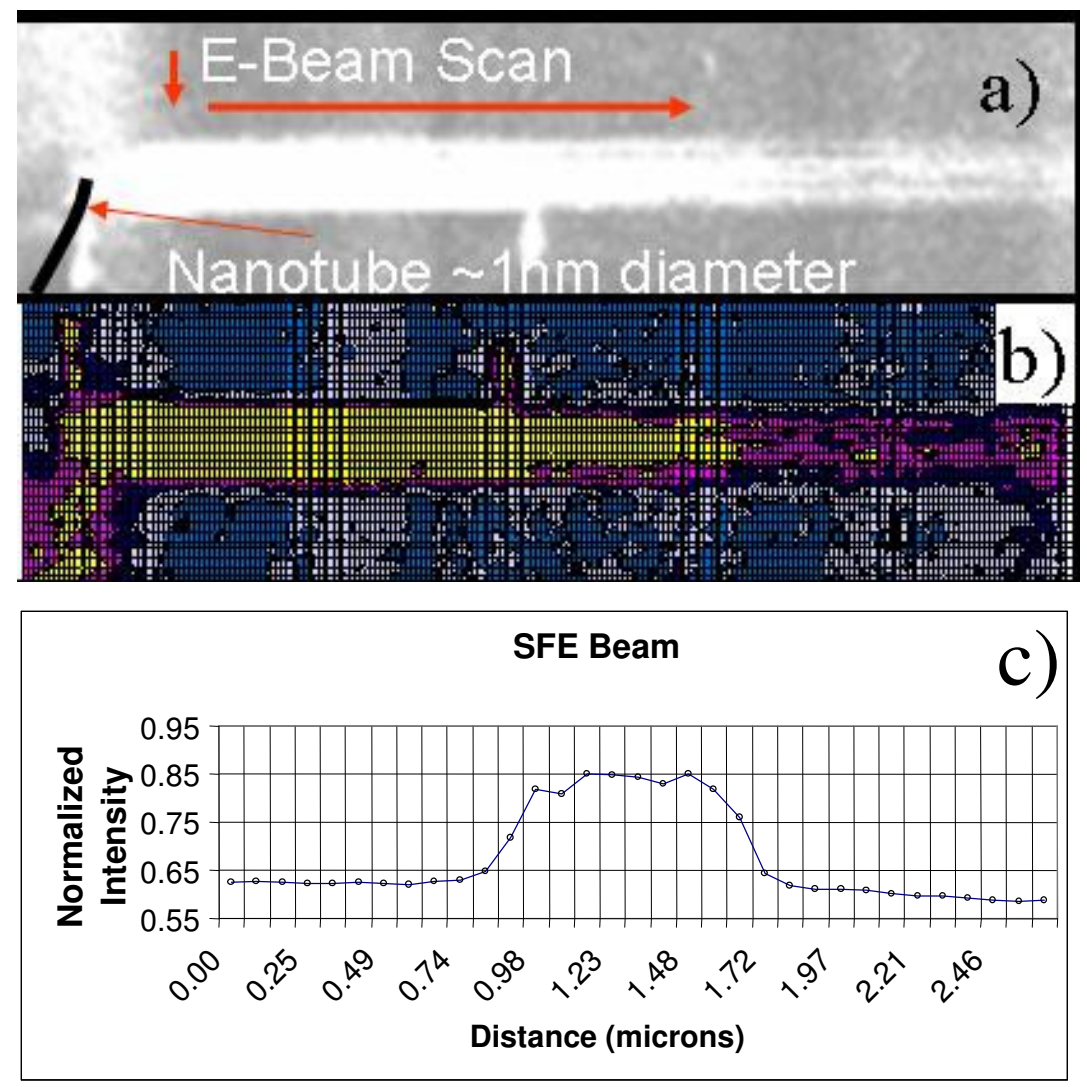

Figure 2.6: a) Zoomed-in image of the SFE spot of Figure 2.3 with the primary beam scanning direction and emitting nanotube position as indicated. b) Pixel intensity map extracted from image. c) Average intensity of each row as a function of distance from the top edge of the SEM image shown in (a). 
The emission current and, as a result, the percentage of electrons captured by the detector increased as the primary beam approached the nanotube, although the plot on Figure 2.6c is truncated due to the saturation of the secondary electron detector. In this example each pixel on the digitized image was $82 \mathrm{~nm}$ x $82 \mathrm{~nm}$ and, as can be seen on Figure 2.6, the interaction area has a radius of approximately $450 \mathrm{~nm}$. In other words, SFE started when the 5-nm-diameter primary beam was as far as $450 \mathrm{~nm}$ from the $\sim 1-\mathrm{nm}$ diameter CNT. This distance is too large for direct interaction of the primary beam electrons and the CNT tip. This indicates that there is some indirect interaction at play. One possibility is that the oxide layer acts as an intermediary to enable this long-range interaction. Below we further investigate this possibility.

When the primary beam impinges on the $\mathrm{SiO}_{2}$ layer, it produces reflected electrons that could escape the surface some distance away from the primary beam. These electrons could potentially be interacting with the nanotube and leading to SFE. In order to verify this hypothesis Monte Carlo simulations of the interaction of the primary beam electrons and the $\mathrm{SiO}_{2}$ layer were performed using the software CASINO [16]. Figure 2.7 shows the electron trajectories for a primary beam energy of $5 \mathrm{keV}$ and a spot size of $5 \mathrm{~nm}$. The penetration depth and radius of the interaction can be observed. 

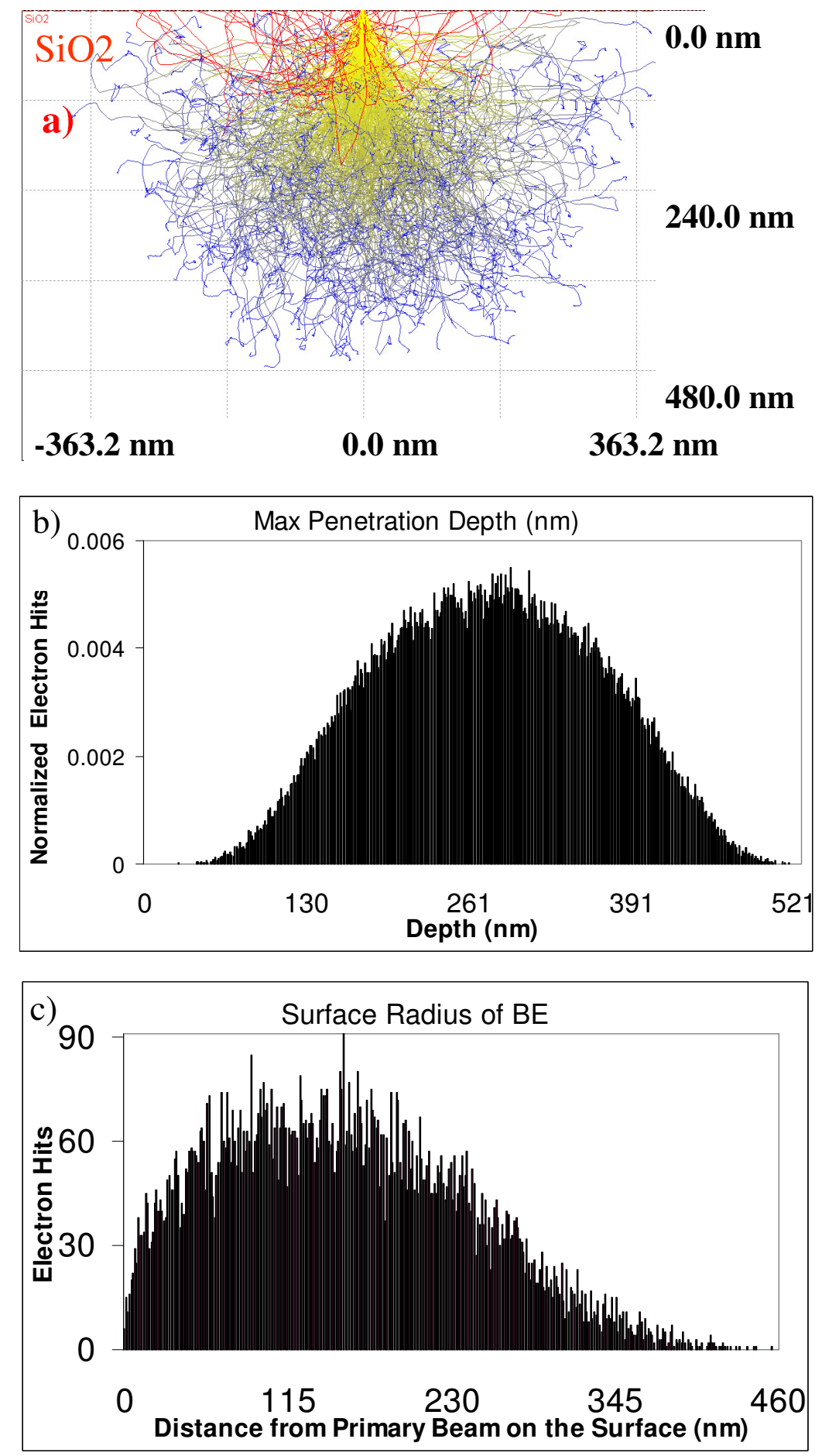

Figure 2.7: a) Electron trajectories calculated with a Monte Carlo simulation of the primary beam interaction with the $\mathrm{SiO}_{2}$ substrate using the program CASINO [16]. The primary beam energy is $5 \mathrm{keV}$ and the spot size $5 \mathrm{~nm} ; 100,000$ electrons were used in the simulation. b) Distribution of electrons as a function of penetration depth into the substrate. The maximum 
depth is $\sim 520 \mathrm{~nm}$. c) Distribution of backscattered electrons (energy greater than $50 \mathrm{eV}$ ) as a function of distance from the primary beam on the substrate surface.

From the figure it is evident that backscattered electrons (BEs) and, as a consequence, secondary electrons (SE) can be reflected from the surface as far as about $480 \mathrm{~nm}$ from the primary beam location. This is consistent with the observation that the interaction seems to occur as far as 450 $\mathrm{nm}$ and provides a possible explanation for the size of the interaction area observed.

Other interaction mechanisms are not necessarily ruled out. An important such mechanism could be the charging of the oxide region around the nanotube tip as a result of irradiation by the primary beam, which could significantly affect the local electric field. For instance, not all of the primary beam charge is reflected and some of it will stay in the substrate. The BEs will also create more SEs as they move through the lattice and some of those SEs generated below the escape depth will charge the surface. The escape depth of SEs for $\mathrm{SiO}_{2}$ is between $10 \mathrm{~nm}$ and 20 $\mathrm{nm}$ [17] and charge will most likely accumulate below this depth in a region with a radius of about $480 \mathrm{~nm}$ around the primary beam for the $5 \mathrm{keV}$ case. Oxide charging will affect the electric field at the tip of the nanotube, potentially assisting in electron emission. An observation that supports that oxide charging is playing an important role is that, as mentioned before, SFE is pervasive: Figures 2.3 and 2.6 show bright streaks that extend as far as 2 msec after the primary beam has swept past the initial emission spot. An estimate of the oxide discharge RC constant indicates that the discharge time is in the order of a few milliseconds. Furthermore, SFE was not observed for a 15-keV primary beam. Monte Carlo simulations of the $15-\mathrm{keV}$ beam (Figure 2.8) show that the penetration depth for this energy is larger than $2 \mu \mathrm{m}$, which is the thickness of the oxide layer over a highly-doped, low resistivity silicon substrate. Therefore, at this energy, a continuous discharge path to the ground exists through the so-called electron beam-induced current (EBIC) and no charging occurs in the dielectric and, consequently, no SFE due to oxide charging. 


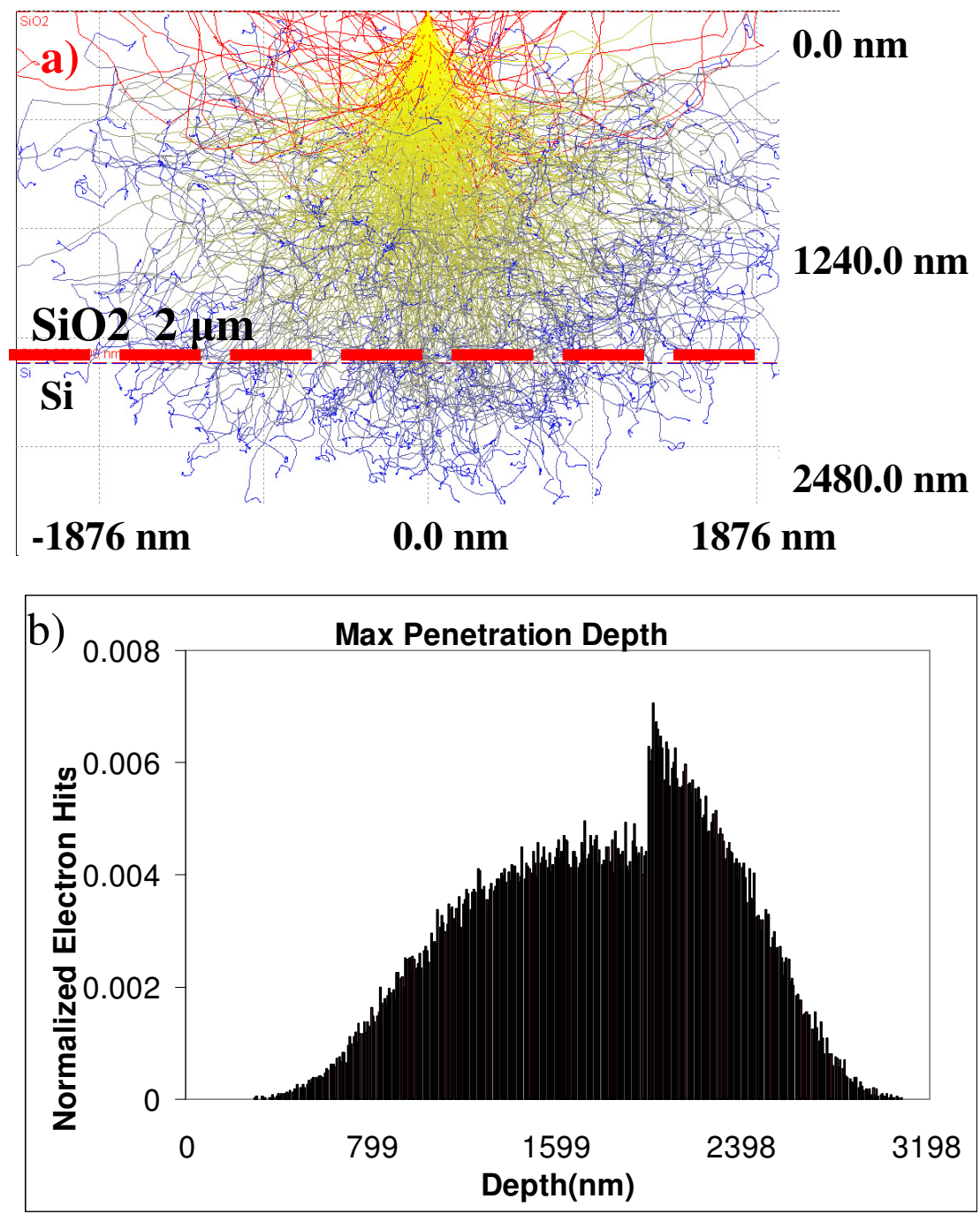

Figure 2.8: a) Electron trajectories calculated with a Monte Carlo simulation of 100000 electrons at $15 \mathrm{keV}$ and $5 \mathrm{~nm}$ spot size and impinging on a $2-\mu \mathrm{m} \mathrm{SiO}_{2}$ layer on a $\mathrm{Si}$ substrate. b)

Distribution of electrons as a function of penetration depth into the $\mathrm{SiO}_{2}$ layer. The large peak corresponds to the $\mathrm{SiO}_{2}$ - Si interface.

In order to further investigate the effect of dielectric charging and separate it from direct interaction of electrons (primary or secondary) with the nanotube tip, experiments were also performed with a different type of device, with an oxide thickness of $400 \mathrm{~nm}$. At this thickness no substrate charging should occur beyond a primary beam energy of $4.2 \mathrm{keV}$ and therefore no SFE caused by charging. Nevertheless, SFE was observed in these devices at $5 \mathrm{keV}$ and even 10 $\mathrm{keV}$ where absolutely no charging should occur. At $5 \mathrm{keV}$ the primary beam is already reaching the silicon substrate inducing currents and cancelling any surface charging (see simulation in Figure 2.7). The fact that no charging was happening was confirmed by zooming in and out of 
the image in the SEM and observing the brightness of the substrate (no dark charging boxes were observed). Similarly, at $10 \mathrm{keV}$ more than $90 \%$ of the electrons will punch through the oxide layer and the surface charging will be zero or even positive. Interestingly, in these cases the SFE peak current recorded was not as large as in the previous experiments (where there was charging). Furthermore, SFE was not pervasive like in those cases. The maximum gain calculated for these devices was $\sim 20$ at $5 \mathrm{keV}$ and the average gain was $\sim 10$. The main stimulating process in this case is believed to be direct interaction of the CNT with the primary beam electrons or secondary electrons. A possible mechanism for such direct interaction is the rise in the nanotube tip energy levels (effect reduction of workfunction) due to the external electron placed inside the nanotube as investigated in [4]. Another interesting point is that the interaction area observed in these cases is not as large as the surface area of BE escape estimated by Monte Carlo simulations. For instance, for the $10 \mathrm{keV}$ case, the radius of this area is as large as a $1.3 \mu \mathrm{m}$. However, the SFE peaks observed indicate a much smaller interaction area with a radius of approximately $150 \mathrm{~nm}$. Nonetheless, similarly to the cases where oxide charging exists, as the beam energy is increased the electron gain decreases and SFE disappears at about $15 \mathrm{keV}$. This is also consistent with the direct interaction mechanism proposed in [4]: higher-energy electrons spend less time in the nanotube. Thus, the resulting rise in nanotube energy levels will also exist for a shorter period of time (for instance $~ 0.01$ fsec for $15-\mathrm{keV}$ electrons passing through a nanotube with a 1-nm diameter perpendicular to it), which may not be sufficient for the emission of other electrons from the nanotube.

In order to further quantify the effect of substrate charging a simulation of the electrostatic field in the device was performed using the Electrostatics module of the software COMSOL Multiphysics. Figure 2.9 shows the model and Figure 2.10 a comparison of the model with an actual device. The model dimensions were chosen in a way so as to closely mimic one of the actual tested devices. The nanotube was modeled as a hollow perfect conductor with a width of 2 $\mathrm{nm}$ and length of $1.7 \mu \mathrm{m}$. Values of 12.1 and 4.2, respectively, were chosen for the relative permittivity of silicon and silicon dioxide (as given by the COMSOL database). A total of approximately 20,000 mesh points were used for the simulation. Figure 2.10 also shows the distribution of the field in this device without any oxide charging.

The ground connection to the silicon substrate is done through a thin oxide layer on the back of the silicon substrate and occasional contamination. Two extreme cases for this contact can be 
considered: zero and infinite resistance. In the former case, the silicon substrate would be fully grounded and at the same potential as the anode. In the latter, it would be a floating conducting plane. The simulations were performed for both cases (Figure 2.10). However, both simulations give field values within the same order of magnitude and lead to the same qualitative conclusions.

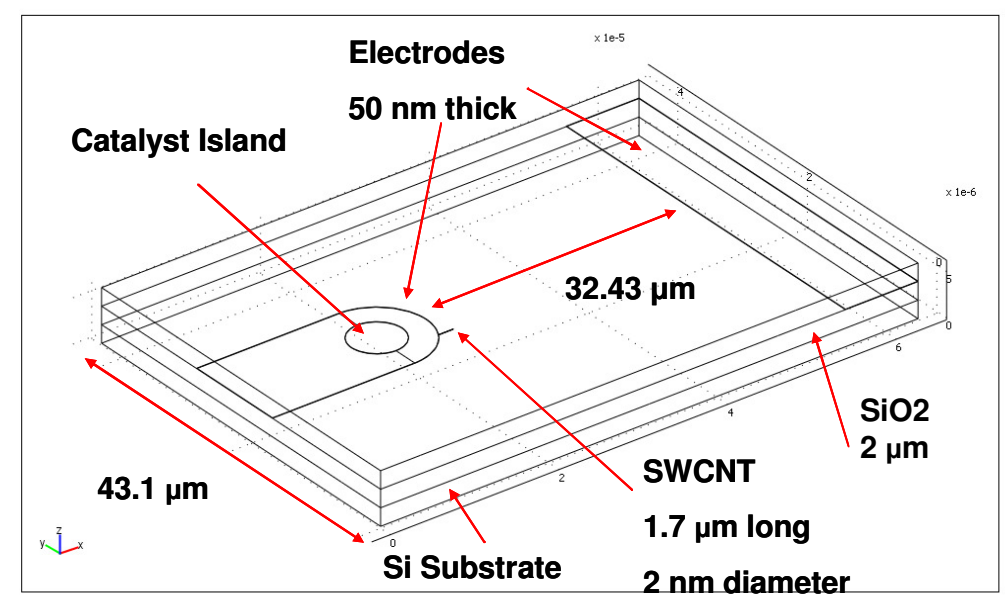

Figure 2.9: Schematic of a model of one of the experimental devices used to simulate the electric field at the tip of the nanotube. The model closely matches the dimensions and materials used in the actual devices. 


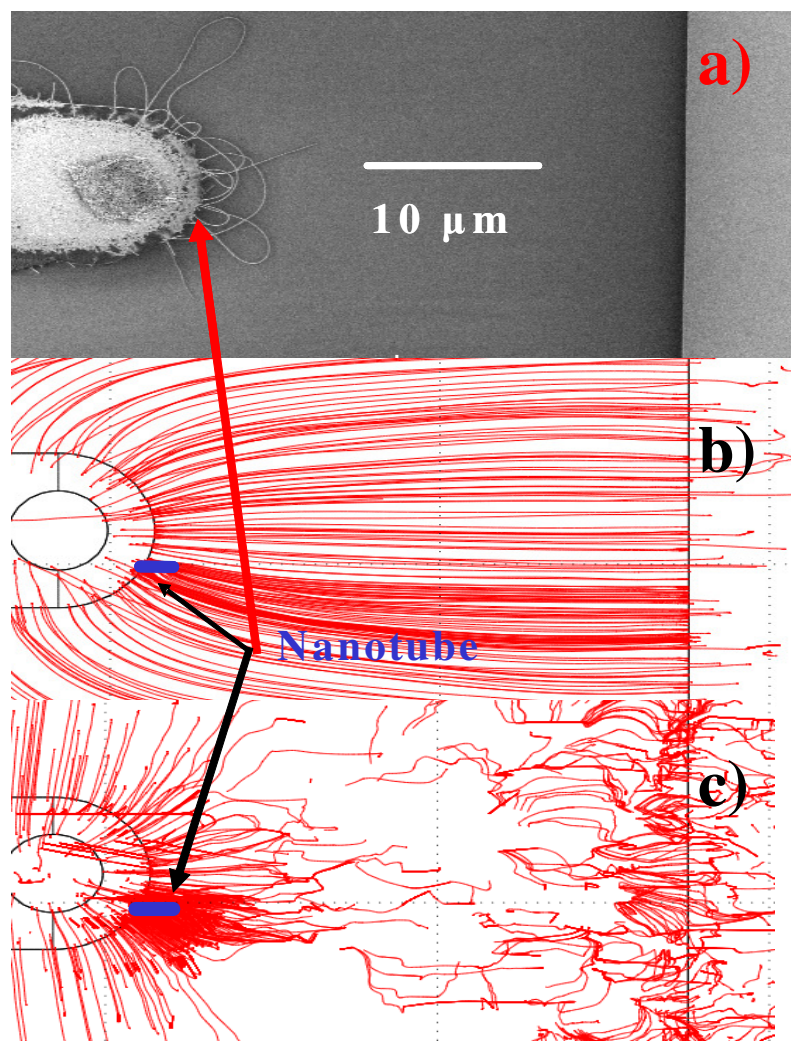

Figure 2.10: SEM image of the device with a floating silicon substrate (a) and top view of the calculated electric field distribution without any substrate charging: without a ground plane (b) and with a ground plane (c). As expected, the image shows a higher electric field flux (higher effective electric field) at the tip of the nanotube. The value of the field at the nanotube tip is comparable in both cases.

In the next step, the oxide charge was calculated as a function of the primary beam energy and current with a Matlab script using the $\mathrm{SiO}_{2}$ backscattered and secondary electron yield coefficients published by Joy [18]. For a detailed description of the calculation see Appendix B or the references $[17,19,20]$. Then, the electric field distribution was simulated again for the device, but this time also including the effect of the dielectric charge. Figure 2.11 shows a volume the size of a "charge pixel" that was moved around the device in the model. This pixel was charged with the value calculated above $\left(-8 \mathrm{C} / \mathrm{m}^{3}\right)$. The pixel is $100 \mathrm{~nm} \times 100 \mathrm{~nm}$ in size and it represents the charging area caused by the primary beam as it scans the device. In the figure, three different positions are shown: the pixel centered below the CNT tip, $100 \mathrm{~nm}$ before the tip and $100 \mathrm{~nm}$ after the tip. 


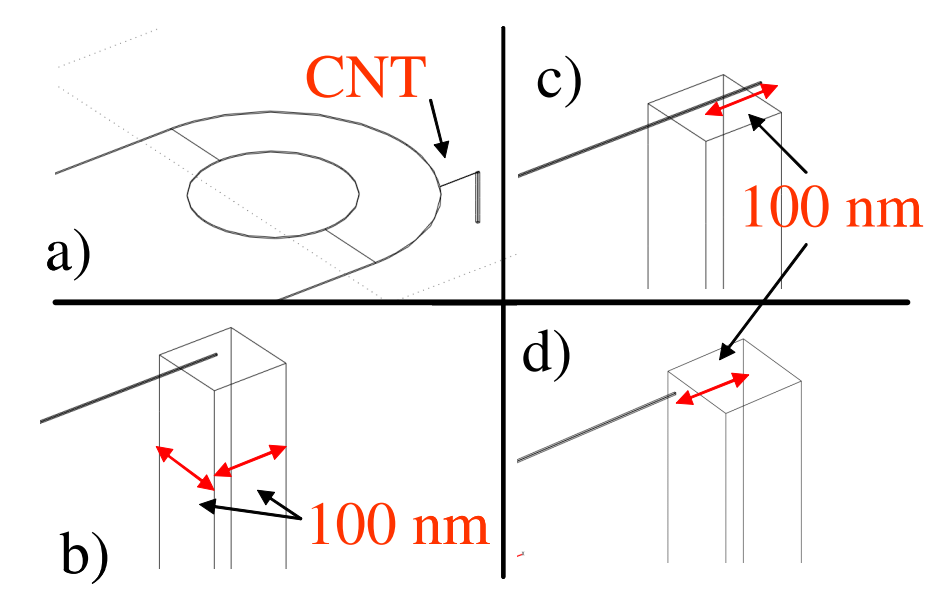

Figure 2.11: a) Schematic of the model showing the cathode electrode with a nanotube and the charge pixel directly below the tip. The charge pixel is $100 \mathrm{~nm} \times 100 \mathrm{~nm}$. b) Charge pixel centered at the CNT tip position. c) Charge pixel $100 \mathrm{~nm}$ before the tip of the nanotube. d) Charge pixel $100 \mathrm{~nm}$ in front of the nanotube tip.

The reason for using a $100 \mathrm{~nm}$ x $100 \mathrm{~nm}$ charge pixel instead of the full interaction area discussed earlier is that the charging electrons are not distributed uniformly over the whole interaction area, but should follow a spatial distribution similar to that of the secondary electrons. In this distribution the charging electron density is highest at the centre and most of the charge will be concentrated within about a 50-nm radius from the centre. The SE distribution has been previously described in the literature [17]. Since according to this distribution most of the charge will be concentrated within a 50-nm radius, a charging pixel size of $100 \mathrm{~nm}$ x $100 \mathrm{~nm}$ (with uniform distribution for simplicity) was used for the simulation. Although this size and shape may seem somewhat arbitrary, note that here we are using them to gain a qualitative, rather than exact quantitative, insight into the effect of oxide charging on electron emission.

On Figure 2.10 we saw the electric field distribution in a device without any surface charging, under an applied bias of $150 \mathrm{~V}$. The field at the nanotube tip was found to be approximately 2 V/Å (typical for field-emission threshold), indicating a field enhancement factor (which we define as the ratio of the field at the nanotube tip to the field at the same location when no nanotube is included in the simulation), of approximately 100. On the other hand, the field enhancement when the substrate directly below the nanotube tip was negatively charged (corresponding to Figure 2.11b) was calculated to increase by 12 times, leading to an enhancement of 1,200. This implies a tremendously high emission current. Of course such a high value will not be reached in practice since electron emission will happen long before this point 
and the charging dynamics will change. Obviously the reality of the interaction is more complex than this static charging model. However, the model provides insight into the strength of this oxide charging mechanism. When the charged pixel was placed $100 \mathrm{~nm}$ behind the tip of the CNT under the CNT body (as in Figure 2.11c), the field enhancement decreased to about 500. This is 5 times the field enhancement without surface charge. When the charge pixel was moved $100 \mathrm{~nm}$ in front of the tip (Figure 2.11d), field enhancement was reduced to 400, namely 4 times the value without surface charging. (The values mentioned above are averages from the two cases simulated, namely with the silicon substrate fully grounded and with the substrate as a floating, conducting plane).

When the charge pixel was placed at any other location, more than $200 \mathrm{~nm}$ away from the CNT, it would actually reduce the field enhancement at the nanotube tip. The worst case found reduced it to 50, namely half of the field enhancement value with no charge at all. This happened when the charge pixel was placed at $1 \mu \mathrm{m}$ directly in front of the CNT. The same value was obtained when the entire substrate was uniformly charged. Since tunneling and, therefore, the electron emission current, are exponentially dependent on the field, these drastic variations in local field because of the action of the primary beam in charging the oxide provide a possible explanation for the observed peaks in SFE current and the high gain obtained. Note that the presence of other nanotubes and defects in a real device would affect the field enhancement factor; however, such effects do not change the qualitative discussion presented here. Other types of mechanisms such as interactions with plasmons, Auger electrons and x-rays may also play a role. Some of these mechanisms have been previously examined by Kasumov et al [21]. Another possible effect could be the enhanced adsorption of residual gases on the nanotube tip that could lead to increased field-emission current $[22,23]$.

It is worth reemphasizing that in a typical experiment, the first SFE peaks obtained are usually quite large, but they gradually decrease in intensity as the primary beam hits the nanotube tip over and over again (Figure 2.4). Given the energy range of the primary electrons, it is unlikely that any direct damage to the tip is caused by the beam. Such damage typically occurs at beam energies beyond $100 \mathrm{keV}$ [24]. However, under the poor vacuum conditions of the experiment, it is expected that the electrostatic deposition of particles or electron-beam induced amorphous carbon deposition on the nanotube tip would gradually deteriorate the tip's sharp structure. Observations of a deformed nanotube tip at the end of the experiments (not shown here) support 
this explanation. This would gradually decrease the emission current and eventually lead to the destruction of the device. So far the damage in all our tested devices has been permanent and no further SFE has been obtained from any device once it has stopped functioning in a particular experiment. Figure 2.12 is the SFE current in a device when a 5-keV primary beam was kept in line scan mode, that is scanning back and forth along a single line, passing through the nanotube tip. The line scan period was $32 \mathrm{msec}$. The experiment was repeated two consecutive times as it can be seen on Figure 2.12, each time for about $10 \mathrm{sec}$ before the beam was slowly moved away from the nanotube. In this configuration the beam hits the nanotube once every 32 msec. Every data point corresponds to the average current for one line scan. The current decreased over time (from the point at about $104 \mathrm{sec}$ in Figure 2.12 to $111 \mathrm{sec}$ ). In the second stimulation (starting at $\sim 122 \mathrm{sec}$ ), the current began from the last value recorded during the first stimulation and continued decreasing. Notice that in Figure 2.4 we have shown the current readings as the beam was scanning the sample at $32 \mathrm{msec}$ per line and the frame consisted of 250 lines. According to the scanning time, the SFE occurred every $8 \mathrm{sec}$. In that case the current peaks also decreased with time.

SFE @ 5keV 32 msec continous line scan

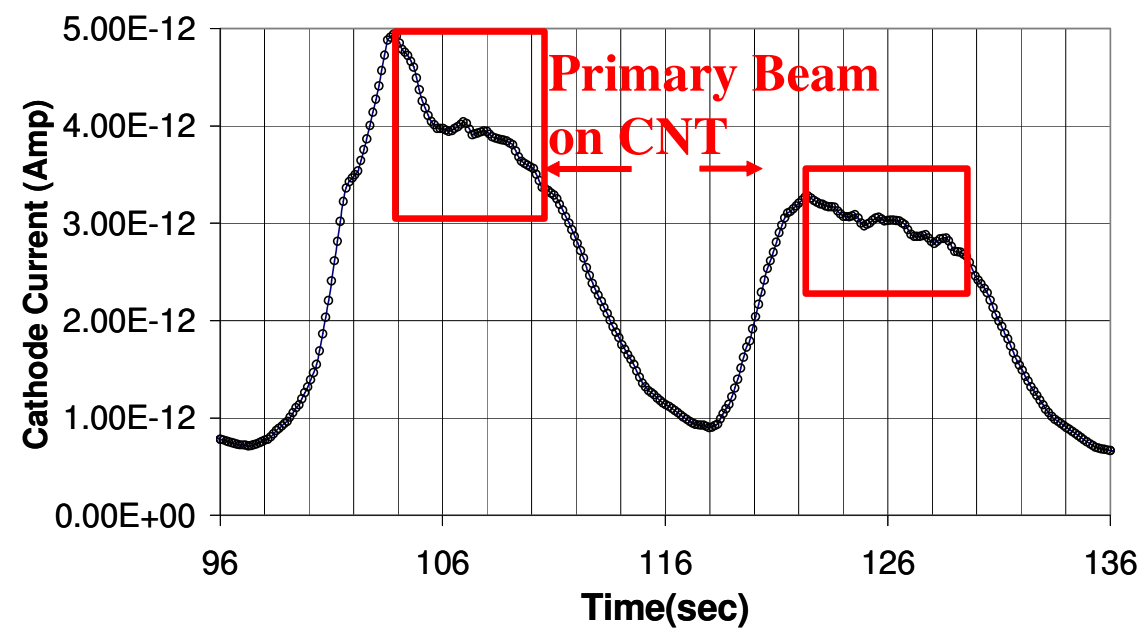

Figure 2.12: SFE with a 5-keV primary beam in line scan (32 msec per line) along a line passing through the nanotube tip. The nanotube tip was being hit every $32 \mathrm{msec}$ by the primary beam. Every data point corresponds to the average current for a line scan. The beam was slowly moved toward the nanotube at time $\sim 100 \mathrm{sec}$ and away starting at approximately time $111 \mathrm{sec}$. It was brought back over the nanotube starting at approximately $118 \mathrm{sec}$ and away at $130 \mathrm{sec}$. 
Finally, a note on the SEM imaging of nanotubes is in order: given the small interaction area of a single-walled carbon nanotube with the primary beam, it has been rather surprising that SWNTs are so readily observable in an SEM. The fact that, as revealed by the present work, the primary beam seems to have both strong direct interaction with the nanotubes, as well as strong interaction with them through the substrate (consistent with previous reports such as that of Kasumov et al [21]), is thus relevant to nanotube electron microscopy in general.

\subsection{Summary and Future Work}

Two interaction mechanisms, namely direct interaction of primary electrons and oxide charging seem to play a role in electron-stimulated field-emission. Direct electron-nanotube interactions seem to account for lower electron gains, in the order of 20-10. Substrate charging, on the other hand, appears to account for much larger gains. In the 5-keV experiment an average gain of approximately 1000 was calculated and this was the result of both types of interactions. The maximum gain obtained in our experiments was 2300. Other mechanisms such as interactions through plasmons, Auger electrons and x-rays may also play a role, but we have not investigated those here.

Regardless of the actual interaction mechanisms behind this effect, the high values of gain could find a use in applications such as vacuum nanotransistors, electron multipliers or detectors with high spatial resolution. The gain of such devices could be improved by the proper choice of dielectric and device geometry. However, more work is needed in order to make such applications a reality. For example, knowledge of the energy distribution of the emitted electrons can provide valuable additional information on the nanoscale interactions. Also experiments in ultra high vacuum may provide more clear readings of SFE and more insight into the reasons for emission decay and much more clear readings of SFE. Additionally, experiments on arrays of nanotubes to possibly obtain higher gains will be interesting.

\subsection{Acknowledgements}

We thank Ali Kashefian for wirebonding the devices. Financial support was provided by the Natural Sciences and Engineering Research Council (NSERC) of Canada (Grant No. 341629-07) 
and the Canada Foundation for Innovation (CFI - Grant No. 13271). We also thank the Stanford University Beckman Cell Sciences Imaging Facility for their generous donation of the Philips 525M scanning electron microscope. J.M.M. also acknowledges support through a fellowship from the Bullitt Environmental Foundation. 


\subsection{Bibliography}

[1] L. A. Chernozatonskii, Y. V. Gulyaev, Z. Y. Kosakovskaya, N. I. Sinitsyn, G. V. Torgashov, Y. F. Zakharchenko, E. A. Fedorov, and V. P. Valchuk, Chemical Physics Letters 233, 63 (1995).

[2] W. A. de Heer, A. Chatelain, and D. Ugarte, Science 270, 1179 (1995).

[3] L. A. Rinzler, A. G. Rinzler, J. H. Hafner, P. Nikolaev, L. Lou, S. G. Kim, D. Tomanek, P. Nordlander, D. T. Colbert, and R. E. Smalley, Science 269, 1550 (1995).

[4] J.-M. Bonard, T. Stockli, O. Noury, and A. Chatelain, Applied Physics Letters 78, 2775, (2001).

[5] Y. Saito and S. Uemura, Carbon 38, 169, (2000).

[6] J.-M. Bonard, H. Kind, T. Stockli and L.-O. Nilsson, Solid-State Electronics 45, 893 (2001).

[7] N. de Jonge, Advances in Imaging \& Electron Physics 156, 2159 (2009).

[8] P. Yaghoobi and A. Nojeh, Modern Physics Letters B 21, 1807 (2007).

[9] A. Nojeh, W.-K. Wong, A. W. Baum, R. F. Pease, and H. Dai, Applied Physics Letters 85, 112, (2004).

[10] A. Nojeh, W.-K. Wong, E. Yieh, R. F. Pease, and H. Dai, Journal of Vacuum Science and Technology B 22, 3124 (2004).

[11] C.-D. Kim, H.-S. Jang, S.-Y. Lee, H.-R. Lee, Y.-S. Roh, I.-S. Rhee, E.-W. Lee, H.-S. Yang, and D.-H. Kim, Nanotechnology 17, 5180, (2006).

[12] A. Nojeh, B. Shan, K. Cho, and R. F. W. Pease, Physical Review Letters 96, 056802, (2006).

[13] E. Pop, D. A. Mann, K. E. Goodson, and H. Dai, Journal of Applied Physics 101, 093710, (2007). 
[14] G. C. Kokkorakis, A. Modinos, and J. P. Xanthakis, Journal of Applied Physics 91, 4580, (2002).

[15] C. Adessi and M. Devel, Phys. Rev. B 65, 075418, (2002).

[16] D. Drouin, A. R. Couture, D. Joly, X. Tastet, V. Aimez, and R. Gauvin, Scanning 29, 92, (2007).

[17] H. Seiler, Journal of Applied Physics 54, R1, (1983).

[18] D. C. Joy, A Database of Electron-Solid Interactions, 2008, http://web.utk.edu/ srcutk/htm/interact.htm.

[19] D. C. Joy and C. S. Joy, Micron 27, 247, (1996).

[20] L. Reimer, (1993), Image formation in Low Voltage Electron Microscopy, Bellingham, WA: SPIE.

[21] Y. A. Kasumov, I. I. Khodos, M. Kociak, and A. Y. Kasumov, Applied Physics Letters 89, 013120, (2006).

[22] K. A. Dean, P. Von Allmen, B.R. Chalamala, Journal of Vacuum Science and Technology B 17, 1959 (1999).

[23] A. Maiti, J. Andzelm, N. Tanpipat, and P. V. Allmen, Physical Review Letters 87, 155502 (2001).

[24] A. V. Krasheninnikov, and F. Banhart, Nature Materials 6, 723, (2007). 


\section{Conclusion and Ongoing/Future Work}

Carbon nanotube-based electron sources show properties superior to traditional sources such as tungsten filaments or $\mathrm{LaB}_{6}$ tips. Particularly, the high brightness, low energy spread and ability to operate at room temperature make them promising candidates for electron-beam technology. Not only vacuum electronics but also microscopy, lithography and manufacturing applications such as electron-beam welding and machining can benefit from the superior properties of carbon nanotube electron emitters. For instance, vacuum tubes made of MWNTs have been prototyped [1]. These tubes showed long life and stable emission (2,000 hours of operation at $30 \mathrm{kV}$ without reduction of current and 10,000 hours of predicted lifetime at normal operating voltages of 1.2 $\mathrm{kV}$ DC). Existing vacuum tubes such as pentodes or more specialized devices such as klystrons can be re-designed based on carbon nanotube electron sources leading to an increase in their performance and opening the door for new applications. The phenomenon of stimulated fieldemission from carbon nanotubes characterized in the present work creates yet another range of technological opportunities: as the basis of a new class of current-controlled current sources, it can form the basis of new devices such as nanoscale vacuum transistors, microscale electron multipliers and high-spatial-resolution detectors. Furthermore, the high electron gains of up to 2300 obtained in the experiments make this effect an excellent candidate for such applications.

Electron stimulated field-emission is a recently reported phenomenon that was first observed on SWNTs when attempting to image field-emission spots with an SEM [2, 3]. During these experiments it was observed that when a nanotube was under external electric field near the threshold of field-emission, an SEM electron beam hitting its tip induced strong electron emission (which was then called stimulated field-emission) from the nanotube. First-principles simulations supported the idea that direct interaction of beam electrons with the nanotube could be a mechanism responsible for this effect [4]. Additionally, stimulated field-emission has also been reported on MWNTs by a different group [5]. By the time of the writing of this thesis the works referred above constitute the state of the art on this topic. During this pioneering work it was observed that stimulated field-emission showed some electron gain. Additionally, the first principle simulations indicated that one electron interacting with the nanotube could lead to 
several electrons being emitted. Verifying that the stimulated field-emission indeed produces some electron gain was what motivated the work leading to this thesis. The work presented in this thesis is the first characterization done of this effect.

During the characterization the high electron gain expected was indeed observed. However, it was noted that some experimental results indicated a different interaction than the direct electron-nanotube interaction. For instance, the emission spots observed in the SEM were larger than what is expected from such an interaction. It was also observed that the emission current continued for several milliseconds after the SEM beam touched the nanotube which is also incompatible with the direct electron beam-nanotube interaction. These observations lead to the idea that a so-called indirect interaction was responsible for a large part of the emission currents measured. The discovery and subsequent analysis of this interaction is, perhaps, my main contribution to this work.

A large part of chapter two is dedicated to explain how substrate charging and electron beam scattering (indirect interaction) can stimulate electron emission from nanotubes and produce very large gains. The analysis is supported by accurate experimental results and well established simulation techniques based on very well known physics (electrostatics and scattering from bulk crystals). This is in my opinion what constitutes the main strength of this thesis. However, the work presented does not provide and in-depth analysis and experimental results to explain the direct electron-nanotube interaction observed by two independent groups $[2,5]$. One of the reasons why this work does not address that question more comprehensively is because this interaction is largely obscured by the indirect interaction and analysing it requires a more sophisticated experimental apparatus than the one used for the characterization. As will be discussed in this chapter, additional experiments have supported the idea of a direct interaction and the high electron gain expected. Additionally, an important part of the ongoing work in our group is to build such a sophisticated experimental apparatus to perform more conclusive experiments. This work is thoroughly described in Appendix A.

In summary, the analysis presented showed two possible interaction mechanisms, namely direct interaction with primary electrons and oxide charging, as responsible for the high electron gain obtained. By carefully choosing the structure (one vs. a group of nanotubes, substrate material and the relative positioning of the nanotube(s) and the substrate), it is conceivable to fabricate 
devices where one specific interaction mechanism is dominant. For instance, direct electronnanotube interactions, which seem to account for electron gains on the order of 10-20, can be used to design electron multipliers where arrays of nanotubes are used as the dynode material. On the other hand, substrate charging, which appears to account for much larger gains - on the order of 1,000 - can be used to fabricate vacuum nano-transistors where the electron emission is stimulated by charging the substrate directly below the nanotube tip.

We suggest two paths for future work on stimulated field-emission. The first is device engineering, where new sensors and devices are designed using the results of the characterization and analysis presented. This empirical path can lead to powerful new applications and it is indeed being pursued: we are working on electron multipliers using arrays of MWNTs. Preliminary experiments show that high gains - on the order of 10,000 - are possible from arrays of MWNTs, and detectors are currently being designed based on a number of such arrays. The second path consists of trying to further understand the fundamental physics of the stimulation mechanisms through more theoretical and experimental work. This can be accomplished with more accurate experiments and through the detailed characterization of different types of nanotubes with, for example, different diameters and chiralities.

The preliminary experiments performed on arrays of MWNTs (nanotube forests) not only showed that the direct interaction is in fact stimulating electron emission with a high gain, but also served as a proof of concept motivating the design and fabrication of detectors based on this effect. As described in chapter two, it was observed that direct interaction accounted for gains of up to 20, which generally decreased as the energy of the SEM electron beam was increased: the maximum gain was obtained for $5-\mathrm{keV}$ primary electrons and almost no gain was observed at primary energies of $15 \mathrm{keV}$ and beyond. This is consistent with the fact that faster stimulating electrons spend less time in the vicinity of the nanotube and are thus expected to interact with the nanotube less effectively. The above observations led us to the idea that if an electron beam irradiated an array of nanotubes, such as a nanotube forest, under applied electric field, an avalanche effect could take place: the slow moving secondary electrons generated through stimulated field-emission from some of the nanotubes could, in turn, stimulate electron emission from other nanotubes in the array and thus create a very large gain. Also, since there is not an insolating substrate, as the nanotube forest is arranged in a free standing configuration, there is not a possibility for the indirect interaction to occur. Figure 3.1 shows a schematic of the 
experimental apparatus, which is the same apparatus described in chapter two, but modified to accommodate nanotube forests. The MWNT forests were grown using chemical vapor deposition on highly doped silicon with a catalyst layer (iron/alumina). The forests were approximately $100 \mu \mathrm{m}$ in length and covered an area of $5 \mathrm{~mm} \times 5 \mathrm{~mm}$. A bias voltage of $\sim 400 \mathrm{~V}$ was applied to an anode placed 0.5 to $1.0 \mathrm{~mm}$ away from the tip of the nanotube forest. The cathode current was measure as the SEM electron beam with a 5-nm spot size and 0.8-pA current scanned across the nanotube forest. Figure 3.2 shows the direction of the scan relative to the nanotube forest.

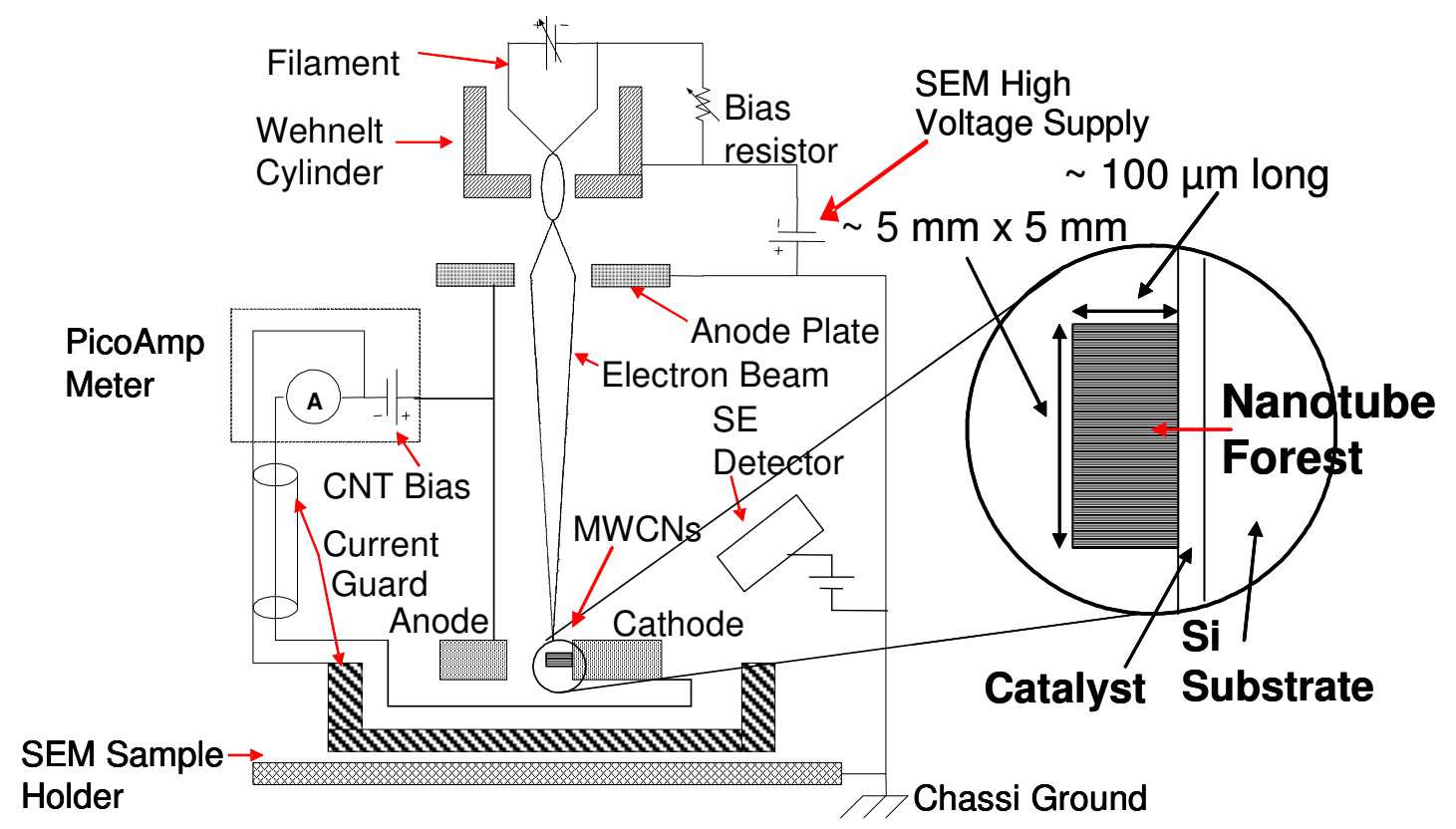

Figure 3.1: The experimental apparatus. The electron beam of the SEM is used to stimulate fieldemission from the nanotubes. The emitted current is measured at the cathode with an electrometer. The nanotube forests are approximately $100 \mu \mathrm{m}$ long and cover an area of about 5 $\mathrm{mm}$ by $5 \mathrm{~mm}$.

The maximum electron gain obtained from a nanotube forest was $\sim 19,000$. Figure 3.2 shows the field-emission current measured as the electron beam scans across the nanotube forest. Since the electron beam is only hitting one side of the forest, the high gain obtained suggests that scattered electrons or secondary electrons generated within the forest may be stimulating emission from nanotubes deeper inside the forest: a scattered electron cloud spreads in the forest, stimulating field-emission as electrons reach the tips of nanotubes well below the side being directly irradiated. The scattered electron cloud is known to have a tear drop shape inside crystals. This 
shape was shown in chapter two for the case of a silicon dioxide substrate (Figures 2.7 and 2.8). Stimulated field-emission current was measured even when the electron beam, which is approximately $5 \mathrm{~nm}$ in diameter, was scanning the body of the top-layer nanotubes several microns away from their tips. This suggests that the cloud of scattered electrons spreads in diameter as it penetrates the forest from the side, consistent with the tear drop shape. We also note that higher currents are stimulated when the beam is scanning around the tip of the nanotubes (see Figure 3.2). This is consistent with our previous experiments on individual nanotubes, where most of stimulated emission happened when the primary beam hit the nanotube tip, leading to a bright spot on the nanotube tip in the SEM image.

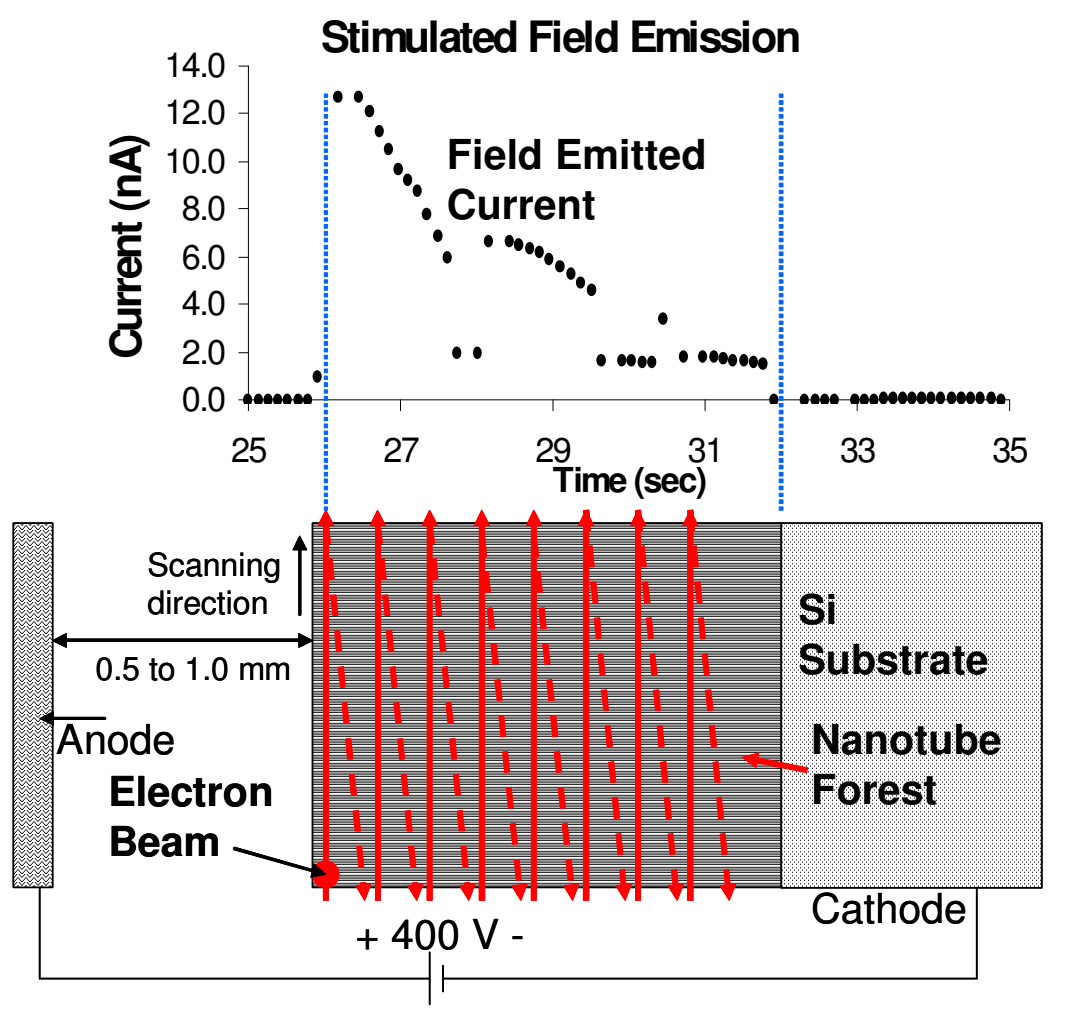

Figure 3.2: Stimulated field-emission from nanotube forest. The maximum gain obtained was 19,000. The arrows on the figure indicate the scanning direction and position of the electron beam. The diagram is aligned with the current vs. time graph to show how the position of the beam is related to the emission current. As the electron beam scans the nanotube forest starting from the tip and advancing towards the body of the nanotubes, the emitted current decreases.

The nanotube forest experiments indicate a direct interaction of the electrons with the nanotubes, as there is not charging, but do not provide any new knowledge about the physical mechanism of 
this interaction. To further understand the fundamental physics of the stimulation mechanisms it is necessary to characterize the nanostructure in a pristine condition and isolated from any other interacting mediums.

Often, to thoroughly characterize nanoscale structures and extract data for fundamental research, "integrated experiments" have to be performed. We define an integrated experiment as one that requires different probing techniques simultaneously. For instance, an integrated stimulated electron emission experiment on a SWNT would require:

(1) Manipulation of a single nanotube with sub-nanometer resolution.

(2) Application of bias and measurement of current with picoAmp resolution or better.

(3) Stimulation by an external electron beam of known energy and the ability to vary the energy and position of the beam with high resolution.

(4) Measurement of the energy of the electrons emitted with sub-eV resolution. Additionally, the most important requirement for such experiments is an ultra clean environment. As discussed in the previous chapter the nanotubes deteriorated during the experiments. This was believed to be due to the material deposition on the nanotube tip during the experiments due to poor vacuum conditions. This hypothesis was further supported by observations of the deformed tip after the experiments. The deposition of material on the nanotubes has also been documented previously [2]. Nanostructures are known to be very susceptible to any type of deposition and adsorption of other molecules as these can change their structure fundamentally. Adsorption, for instance, has been shown to decrease field-emission current from nanotubes [6]. The vacuum level used for the experiments described in chapter two was $\sim 10^{-5}$ Torr. This vacuum level translates to approximately $\sim 3.5 * 10^{10} \mathrm{~cm}^{-3} \mathrm{~N}_{2}$ molecules with a mean free path of $\sim 5 * 10^{3} \mathrm{~cm}$ and an average time of 2.5 seconds for the formation of a monolayer on an ideal, perfectly flat and clean surface of a non-reactive material. The deposition of material increases when the nanostructures are biased due to ionization of neighbouring molecules. It also increases through electron beam-induced deposition when the primary beam of the microscope irradiates the nanotube. In contrast an ultra-high vacuum (UHV) environment with a pressure of $1 * 10^{-12}$ Torr has approximately $\sim 3.5 * 10^{4} \mathrm{~cm}^{-3} \mathrm{~N}_{2}$ molecules with a mean free path of $\sim 5 * 10^{9} \mathrm{~cm}$ and an average time for the formation of a monolayer of $\sim 2.5 * 10^{6}$ seconds [7]. 
These numbers indicate the obvious advantage, if not necessity, of using a UHV system to perform experiments on nanostructures. In parallel with the characterization work already described, a versatile UHV apparatus has been designed and assembled. This equipment will enable more detailed SFE characterization, as well as a host of other experiments on various nanostructures. Appendix A describes this system in detail. 


\subsection{Bibliography}

[1] Y. Saito, K. Hamaguchi, S. Uemura, K. Uchida, Y. Tasaka, F. Ikazaki, M. Yumura, A. Kasuya, and Y. Nishina, Applied. Physics A, 67, 95, (1998).

[2] A. Nojeh, W.-K. Wong, A. W. Baum, R. F. Pease, and H. Dai, Applied Physics Letters 85, 112 (2004).

[3] A. Nojeh, W.-K. Wong, E. Yieh, R. F. Pease, and H. Dai, Journal of Vacuum Science and Technology B 22, 3124 (2004).

[4] A. Nojeh, B. Shan, K. Cho, and R. F. W. Pease, Physical Review Letters 96, 056802 (2006).

[5] C. D. Kim, H. S. Jang, S. Y. Lee, H. R. Lee, Y. S. Roh, I.-S. Rhee, E. W. Lee, H. S. Yang, and D. H. Kim, Nanotechnology 17, 5180 (2006).

[6] S. Suzuki, C. Bower, Y. Watanabe, and O. Zhou, Applied Physics Letters, 76:4007, (2000).

[7] J.H Moore, C. Davis, and M. Coplan, (2002), Building Scientific Apparatus, Massachutsetts: Westview Press. 


\section{Appendices}

\section{A NanoLab: Ultra-High Vacuum Apparatus for Nanostructure Research}

\section{A.1 Introduction}

An ultra-high vacuum (UHV) system developed to perform integrated experiments on nanoscale structures (NanoLab) is described here. An integrated experiment can be defined as an experiment where various probing techniques are used simultaneously. For instance, with this system it is possible to excite a nanostructure with an energetic electron beam, several photon beams and electrical bias while measuring scattered electron energy at various angles, and current and voltages across the device. All of this can be performed simultaneously while the sample is manipulated with sub-nanometer resolution and imaged. The ultra-high vacuum feature provides a clean environment that is essential to preserve the nanostructures over extended periods of time and allow systematic and repeatable experiments. This equipment was entirely designed and built by our group, with the author of the thesis being the leader and performing the majority of the work. Off-the-shelf parts were used as much as possible, although a significant number of components were custom-made.

\section{A.2 Overview of the Apparatus}

Schematic diagrams of the system are shown in Figures A.1 and A.2 where the main components are labelled. Actual pictures of the equipment during assembly are shown in the Figures A.3 to A.6. The system is more easily described by dividing it into three subsystems:

- UHV module and supporting frame

- Sample transfer and manipulation module

- Probes and detectors 
In addition to these three subsystems, auxiliary tools and procedures for system assembly have been designed/created and will be described below.

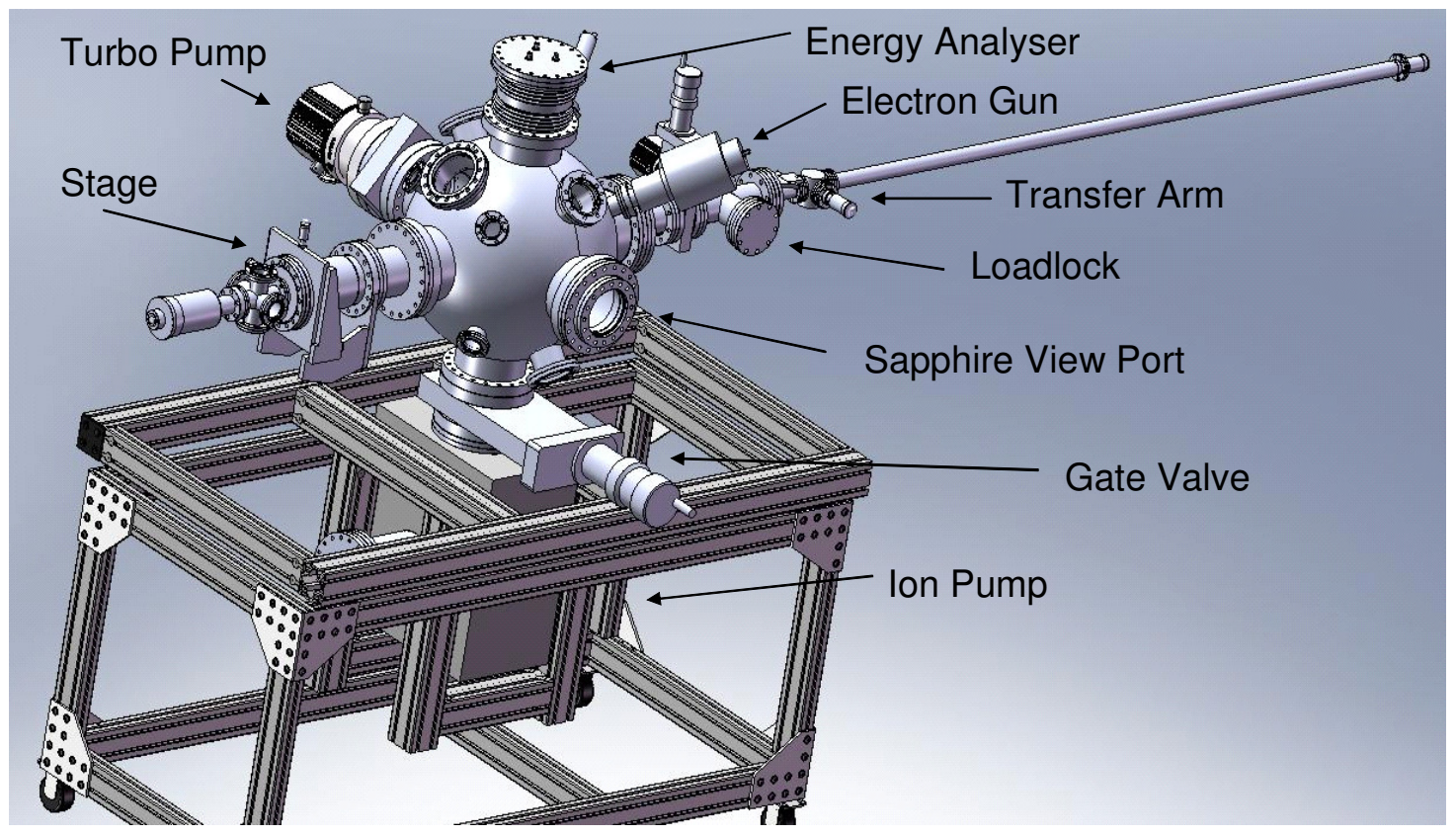

Figure A.1: Schematic diagram of NanoLab. The main components are labelled in this frontisometric view. Hoses, wires and electronic controllers are not included in the diagram. Ports that are not populated are covered with blanks not shown in the schematic.

\section{A.2.1 UHV System and Supporting Frame}

The UHV module consists of two interconnected chambers and a pumping system to evacuate the gases from these chambers. The main chamber is an 18" diameter spherical chamber made of grade 304 stainless steel and fitted with several access ports. This chamber is interconnected to a smaller chamber used as a loadlock by a transfer system and an isolating gate valve. All the connections between elements exposed to the UHV environments are made via ConFlat ${ }^{\circledR}$ flanges. These flanges are commonly used in UHV systems and have a knife edge to extrude a copper gasket and in this way obtain a tight seal. All the ports in both chambers are confocal (all of them point to the center of the chamber). The 304 stainless steel has $11 \%$ of chromium by mass, which makes it not only oxidation resistant but also limits the amount of hydrogen that diffuses through the metal walls into the vacuum. The chromium forms a passivation layer of 
chromium (III) oxide $\left(\mathrm{Cr}_{2} \mathrm{O}_{3}\right)$ when exposed to oxygen. This passivation layer acts as a barrier to hydrogen. This is important as hydrogen is the main residual gas in any UHV system. The pumping system consists of a scroll pump (Varian SH110), two turbomolecular pumps (Varian Navigator Turbo V301 and V81) and an ion pump (Varian VacIon plus 500 combination pump). The scroll pump is a dry pump (there is no oil in contact with the gases being evacuated) and is used for initial evacuation (roughing) from atmosphere down to a pressure of approximately $1 * 10^{-4}$ Torr and for backing the turbomolecular pumps when the chamber is below that pressure. This pump is connected to the two turbo pumps through stainless steel flexible hoses and a system of valves to isolate them as needed. The two turbomolecular pumps are used to pump the system down to approximately $\sim 1 * 10^{-9}$ Torr, that is in the high vacuum (HV) range. TheV301 pump, shown in Figures 1 and 3, has a pumping speed of $2801 / \mathrm{sec}$ for $\mathrm{N}_{2}$ and is attached directly to the main chamber through a gate valve. The smaller V81 pump has a pumping speed of 77 $1 / \mathrm{sec}$ for $\mathrm{N}_{2}$ and is attached to the loadlock. As mentioned this chamber can be fully isolated from the main chamber by another gate valve. The ion pump has a pumping speed of $720 \mathrm{l} / \mathrm{sec}$ for $\mathrm{N}_{2}$ and is used to reach UHV pressures $\left(10^{-10}-10^{-11}\right.$ Torr $)$. It is attached to the chamber by a gate valve and has an extra port for the addition of a titanium sublimation pump in the case that higher vacuum levels are required (in the order of $1 * 10^{-12}$ Torr). 


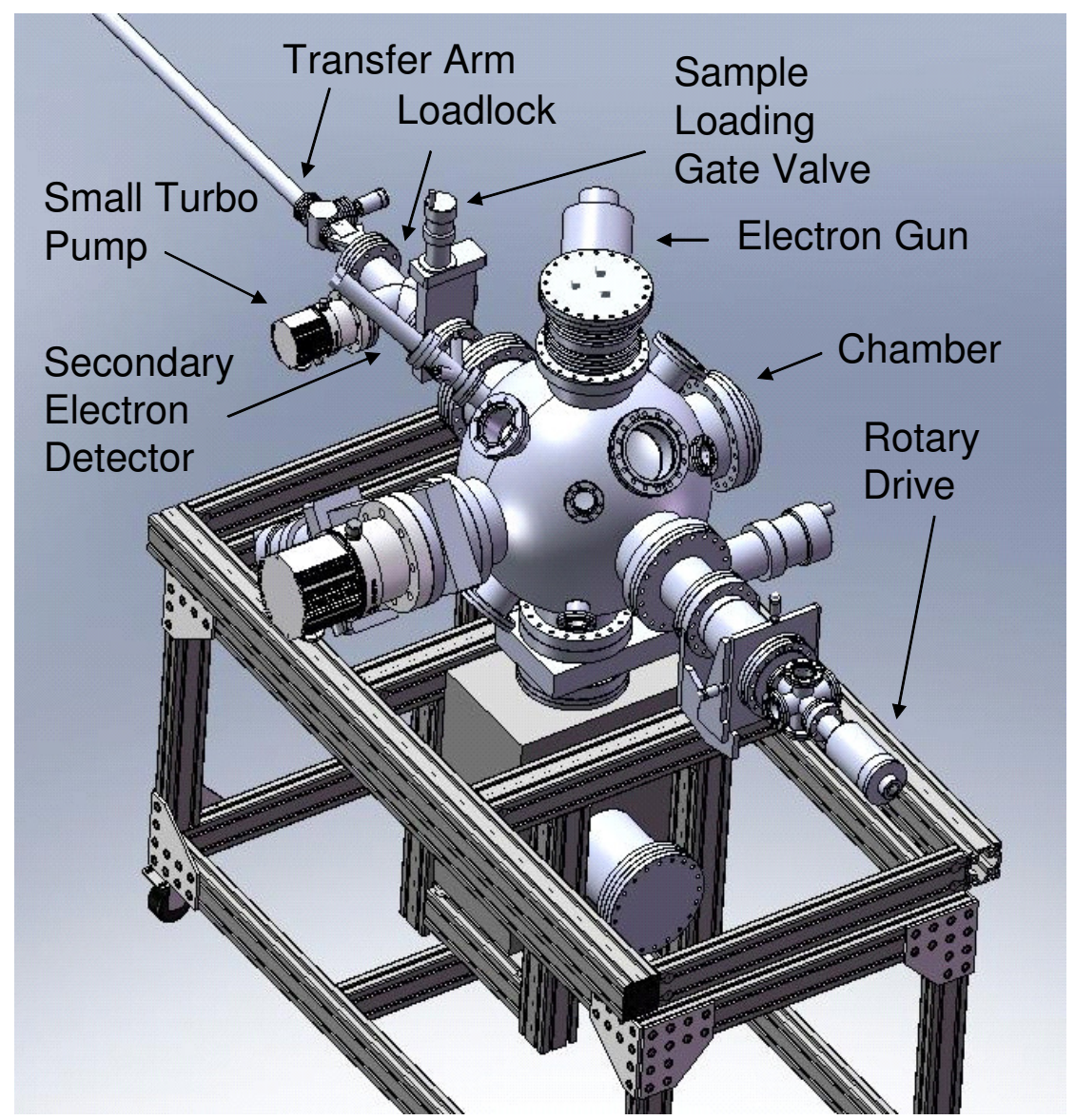

Figure A.2: Schematic diagram of NanoLab. The main components are labelled in this backisometric view. Hoses, wires and electronic controllers are not included in the diagram. Ports that are not populated are covered with blanks not shown in the schematic 


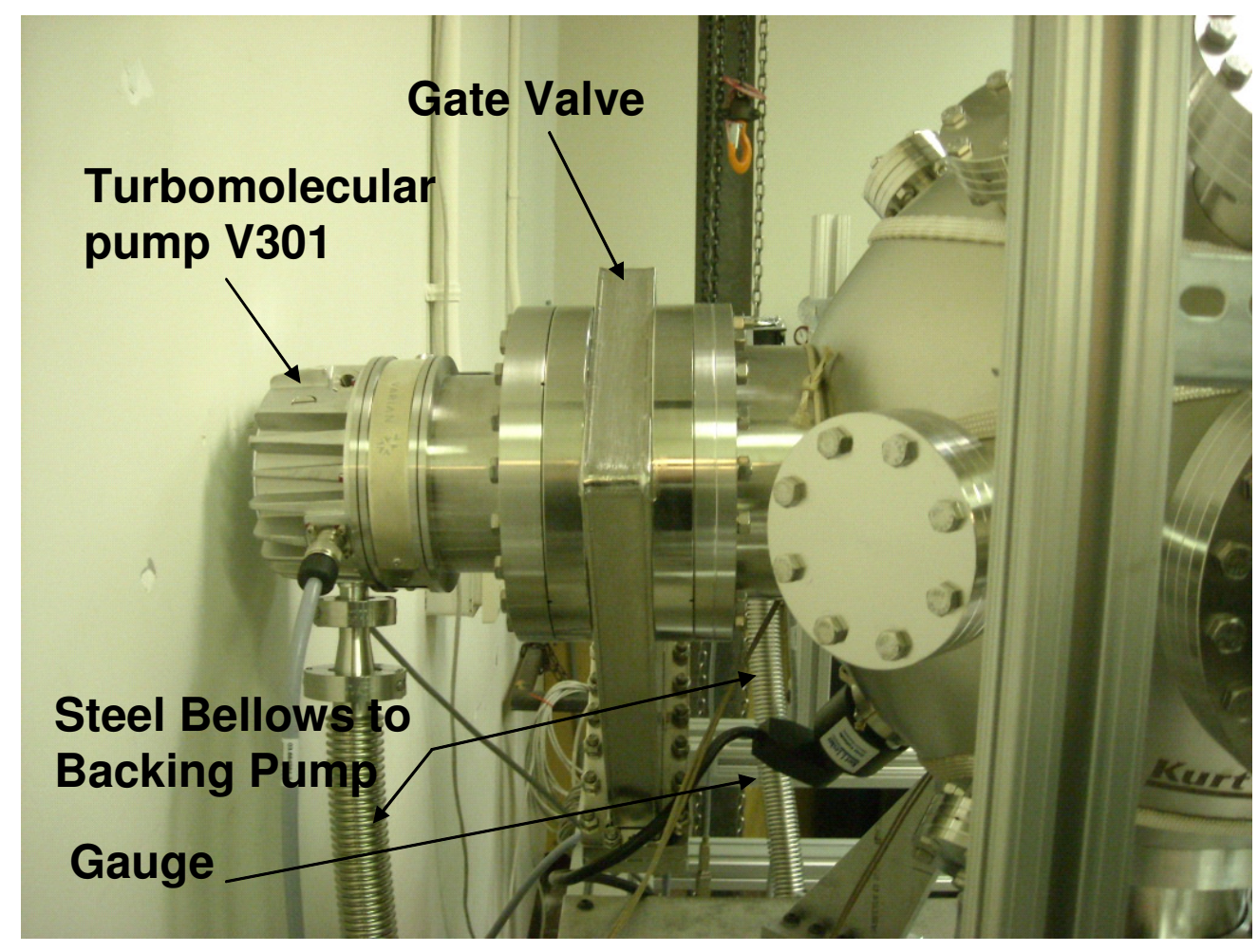

Figure A.3: Large turbomolecular pump V301 and isolating gate valve. The stainless steel hose bellows connecting the turbomolecular pumps and the scroll pump are indicated as well as the Piranni gauge used to measure the vacuum level of the main chamber.

To measure the vacuum level a shielded Piranni gauge has been attached to the main chamber. Additionally, the ion pump provides vacuum measurement feedback. The main chamber can be flushed with nitrogen through an ultra fine leak valve to bring it out of vacuum (venting). The Piranni gauge is shown in Figures A.3 and A.4 and the leak valve in Figure A.4. The system has been designed to reach a vacuum level of $\sim 1 * 10^{-11}$ Torr. At this level of vacuum the surface metal outgassing (release of gas from metal surfaces) becomes comparable to the pumping speeds. In order to reduce metal outgassing the chamber has been wrapped with electric heating tape for baking shown in Figure A.6. During the initial pump down sequence, the scroll pump is first used to bring the system from atmosphere to $\sim 1 * 10^{-4}$ Torr. Once the system reaches this pressure the large tubomolecular pump V301 is started and the system is pumped down to $1 * 10^{-9}$ Torr. The chamber is then baked at $150^{\circ} \mathrm{C}$ for a few days. The temperature is set using a 700-W variac and thermocouple sensors. This procedure increases the rate of outgassing from the metal surfaces and, consequently, the pressure in the main chamber. Most of the gas leakage into the chamber comes from the bulk of the metal. Additionally, a very small amount of the 
smallest-molecule gases such as hydrogen and helium diffuses across the chamber walls. After a few days of baking and pumping down with the turbomolecular pump, the system is finally allowed to cool down to room temperature. At this point the gas inside the bulk of the metals has been completely extracted and outgassing rate decreases. When the system reaches room temperature and a pressure of $\sim 1 * 10^{-9}$ Torr the pumping capability is increased by turning the ion pump on and valving the turbo pump off shortly afterward. The final pressure is dictated by the balance between the diffusion of hydrogen and helium though the chamber walls, a small level of outgassing and the pumping speed for these particular gases. By following this sequence it is possible to reach pressures on the order of $\sim 1 * 10^{-11}$ Torr.

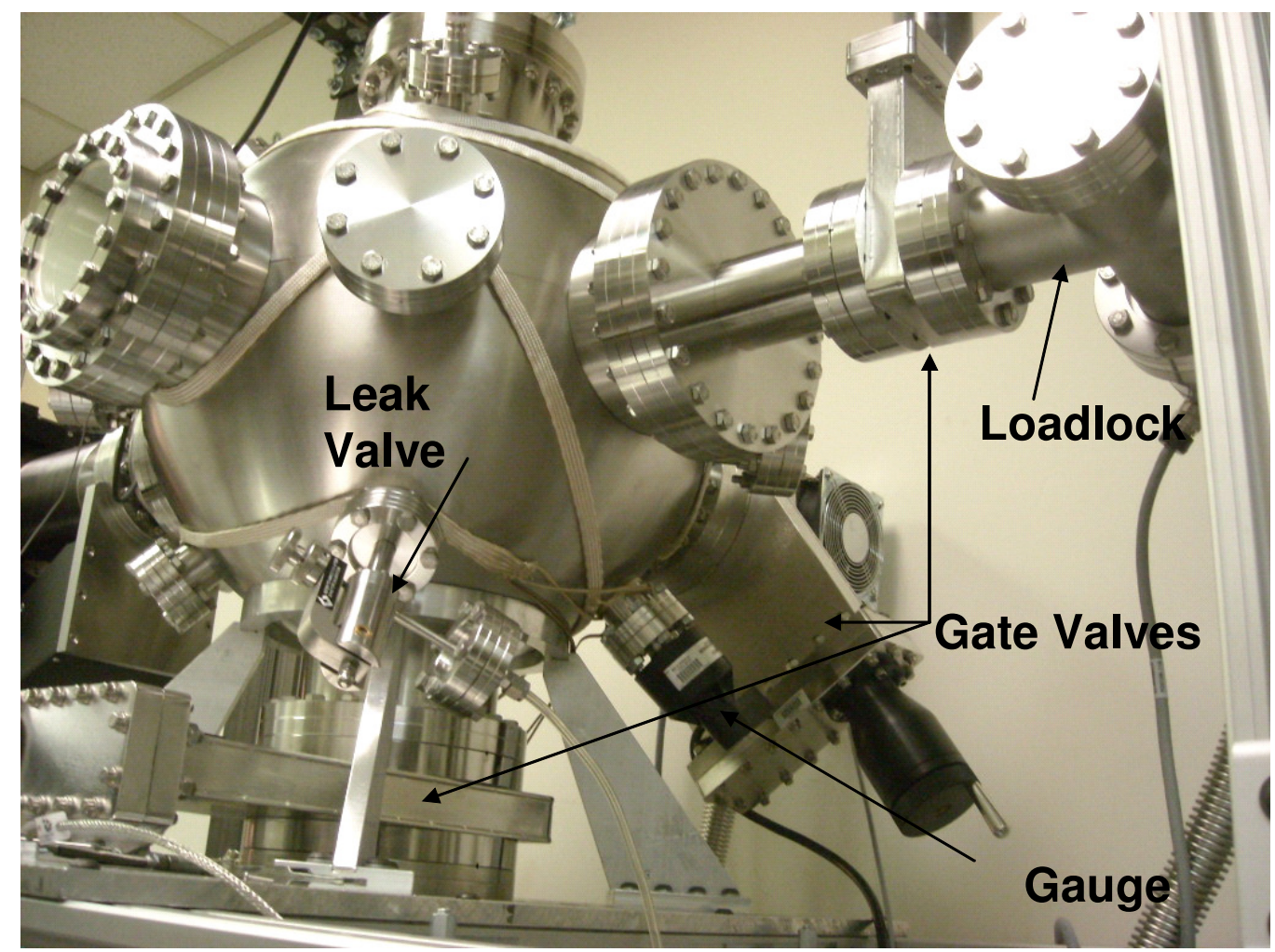

Figure A.4: Piranni gauge and leak valve to control pressure in the main chamber. The gate valves isolate the main chamber from the loadlock chamber, large turbomolecular pump V301 and ion pump.

Once the system is at UHV pressures, loading and unloading of samples is done through the loadlock. The loadlock with the sample in it is pumped down using the scroll pump and the small turbomolecular pump, V81. These two pumps can bring the loadlock down to a pressure of $1 * 10^{-9}$ Torr. To load the sample the pressures in the main chamber and loadlock have to be equalized. This can be accomplished by disconnecting the ion pump and then flushing the main 
chamber with $\mathrm{N}_{2}$. The amount of $\mathrm{N}_{2}$ allowed to leak into the main chamber can be controlled by monitoring the pressure with the Piranni gauge. When a pressure of $\sim 10^{-8}$ Torr is reached the large turbomolecular pump is brought in. After the main chamber has reached an equilibrium pressure equal to that of the loadlock, the loadlock gate valve, shown in Figures A.4 and A.5, can be opened slowly and the sample transferred from the loadlock to the main chamber. Figure A.5 is a picture of the loadlock, gate valve and transfer arm configuration.

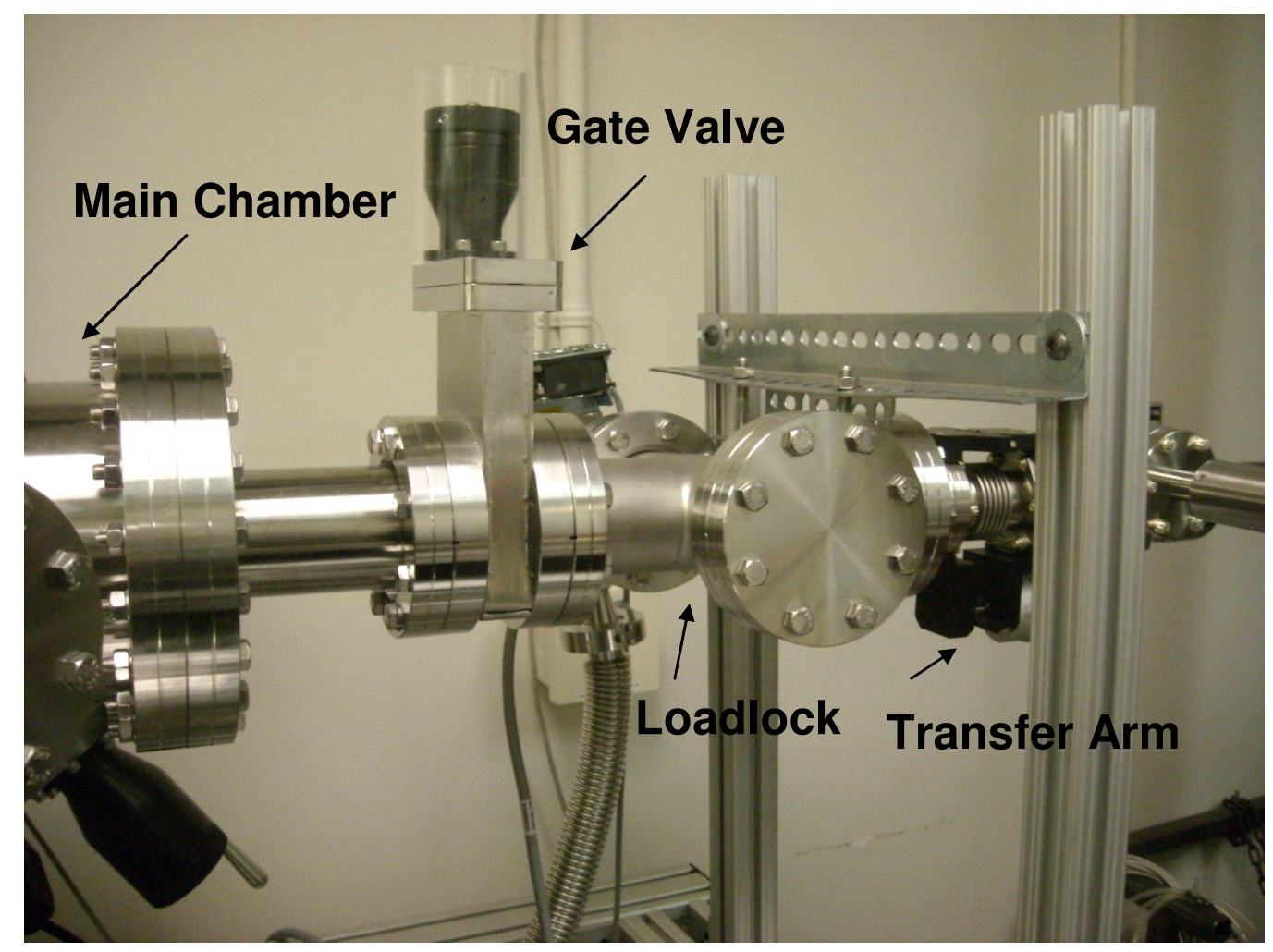

Figure A.5: Loadlock, gate valve and transfer arm configuration. The gate valve isolates the loadlock for sample loading/unloading while the main chamber is held at low pressure. A small turbomolecular pump V81 (behind the loadlock) is used to evacuate the loadlock. The transfer arm transfers the sample into the main chamber where it is placed on a stage for experiments.

To support the whole system a modular frame has been designed and built using aluminium extrusions. The design is such that future extensions to the system can be added easily. The full system weighs approximately $\sim 100 \mathrm{lb}$ (including the frame). The frame has been designed to support this weight with a large safety factor. The frame has been fitted with heavy-duty casters that allow ease of transport in the lab. To reduce vibrations during experiments two sets of shock and vibration absorbers have been used. The first set consists of four elastomeric shock 
absorbers that allow for levelling the frame horizontally. This set of vibration absorbers reduces low-frequency vibrations coming from the lab floor and the building. The second set is composed of four wire-rope shock absorbers (IDC SB16), shown in Figure A.6, that reduce high-frequency vibrations. The shock absorbers dampen any vibration generated from the turbomolecular pumps which spin at 50,000 and 80,000 RPM. It additionally absorbs shocks that could occur by impacts in the building. Figure A.6 shows two of the wire rope shock absorbers and the frame. The scroll pump is decoupled from the main frame and connected to the turbomolecular pumps via flexible stainless steel bellows, visible in Figures A.3 and A.5, which minimize vibration transfer. During sensitive experiments the turbomolecular and mechanical pumps are turned off and only the ion pump is on, which does not have any moving part.

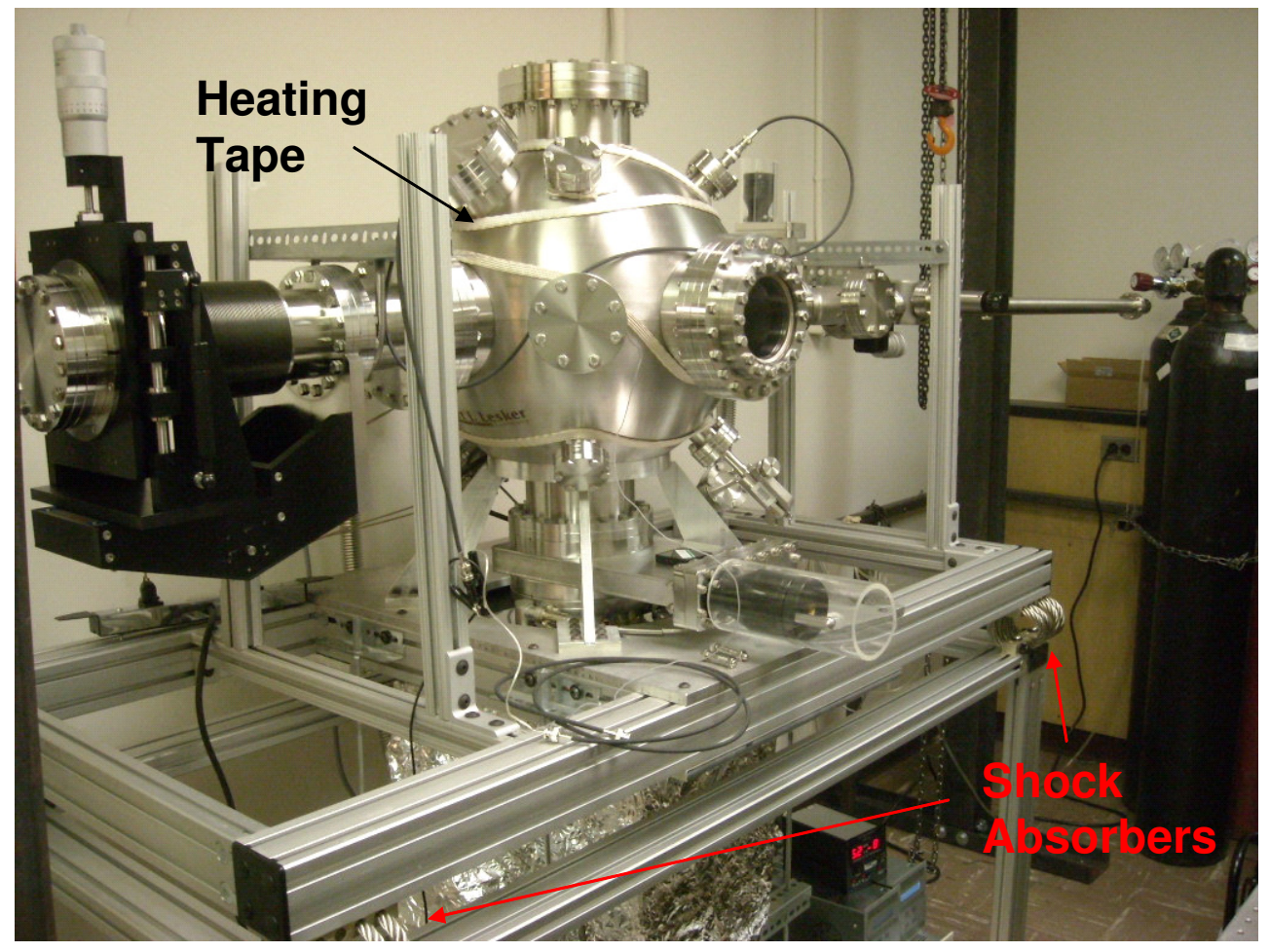

Figure A.6: UHV system and modular frame. The heating tape used to bake the main chamber is visible, as well as the wire-rope shock absorbers.

\section{A.2.2 Sample Transfer and Manipulation}

In order to be able to perform integrated experiments on nanostructures, an insulated, UHVcompatible sample holder has been designed. It is desirable that such a holder accommodate 
various sizes of specimens and a variety of requirements. The sample holder attaches to a stage inside the chamber where it can be moved during experiments. It can hold the nanostructure substrate or a nanomanipulator with the nanostructure substrate on it. The holder is made of Macor ceramic and is equipped with electrical connections for applying bias, measuring current and voltage across the nanostructure, and operating the nanomanipulator. The sample holder is transferred from the loadlock chamber to the stage centered in the middle of the main chamber, where electron and optical beams converge. The transfer is done with a 30" rack and pinion liner transfer arm custom designed and manufactured by Johnsen Ultravac. The arm is attached to the loadlock; once the sample holder is placed on and secured to the stage, the arm retrieves to the loadlock where it is isolated from the main chamber via the loadlock gate valve. Also all the electrical connections are made through the stage. These include a 9-pin, UHV-compatible, Dsubminiature-type connector and four UHV-compatible banana plug connectors for a total of 13 independent connections plus a chassi ground through the body of the chamber. The stage has two additional ports to add more feedthroughs and further connections such as optical fibers, additional sensors such as thermocouples or heating elements or more electrical connections. The stage provides sample manipulation in the three axes with a $0.5-\mu \mathrm{m}$ resolution and $5 \mathrm{~cm}$ of range. It also provides rotation around the arm axis with 1 degree resolution and 360 degrees range through a magnetically coupled rotary drive. The 9-pin D-subminiature-type connector can be used to control a nanomanipulator that fits on the sample holder. Our Attocube ANSxyz 100 is a piezo-driven, UHV-compatible, 3-axis nanomanipulator with a range of $40 \mu \mathrm{m}$ x $40 \mu \mathrm{m}$ x 24 $\mu \mathrm{m}$ and sub-nanometer resolution. All the cables inside the stage are insulated with Kapton and rated for $2500 \mathrm{~V} \mathrm{DC}$. The wires are rated for currents up to $5 \mathrm{~A}$.

\section{A.2.3 Probes and Detectors}

The system is being fitted with an electron gun, a sapphire view port, a cylindrical energy analyser chamber and a secondary electron detector. The electron gun (Staib Instruments HTC 8540.994000 ) is a thermionic emission gun and generates electron beams from 0.0 to $30 \mathrm{keV}$ with a focus spot size of $\sim 100 \mu \mathrm{m}$. It is fitted with electrostatic optics which allow the control of the spot size and beam deflection. Smaller spot sizes can be reached with additional electromagnetic lenses (not built, yet). The 4.5" sapphire view port transmits up to $70 \%$ UV light. It is placed at 90 degrees from the cylindrical energy analyser. Photon beams can be directed to 
the sample through this view port. The cylindrical energy analyser (Physical Electronics Industries Model 15-250) consists of three energy analysers in series: one hemispherical retarding system and two cylindrical mirror analysers. It is meant primarily for high-resolution energy analysis of electrons excited by soft x-rays or by photons in the UV range. The unit enables high-resolution Auger electron spectroscopy when used with the electron gun. In this case the retarding analyser is not used and the electrons are allowed to enter the cylindrical mirror devices with their initial energy. The absolute energy resolution of the electrons is proportional to their initial kinetic energy when emitted from the sample. The resolution in this mode is $0.7 \%$ of the absolute energy. The analyser is mounted through a stainless bellow and linear translator system, which allows for the control of the distance with the sample and retrieval when it is not in use. Finally, the secondary electron detector (Staib Instruments SCI 38) is composed of a scintillator, photomultipler tube, video preamplifier and control units. The detector has a gain of $3.0^{*} 10^{6}$, which can be used for the detection of very weak signals. The output of the detector can be used directly in a video monitor for image formation.

Additionally, the stage has electric coaxial feedthroughs isolated from the chamber body. These connections can be used to measure currents with femto-amp resolution by applying a guard bias on the outer shield of the connector. This bias reduces leakage currents. The inner conductors end in banana plugs in the stage and sample holder. The four banana plugs in the sample holder can be used to implement four-point- probe conductivity measurements in the nanostructures for maximum accuracy.

\section{A.2.4 Auxiliary Equipment}

In order to assemble the UHV system every component that is in contact with the vacuum environment has to be thoroughly cleaned. The following cleaning procedure was developed and followed during assembly:

- Examine all components for damage. All vacuum surfaces shall be polished and free of cracks, pilling, indentations or defects of any kind. Extreme care shall be taken to protect the knife sealing edges and sealing surfaces. Any scratch, pit, inclusion or other defect is not acceptable. 
- Inspect all components to identify areas which may trap contamination. Small and/or blind holes, re-entrant volumes and crevices are examples of areas that must be thoroughly examined and cleaned. Any blind-threaded holes should use vacuumcompatible screws which are perforated for gas evacuation

- Remove all debris and gross contamination by blasting with high-pressure nitrogen and washing and rinsing with any general-purpose solvent. Deposited surface films can be stripped with alumina in water.

- Wash all surfaces with a high-pressure hot water jet $\left(\sim 80^{\circ} \mathrm{C}\right)$, using a mild alkaline nonetch sodium hydroxide detergent with a $\mathrm{pH}=9.5$ to 11 such as $0.2 \%$ (by weight) Alconox ${ }^{\circledR}(\mathrm{pH}=9.5)$.

- Wash thoroughly with a high pressure hot water jet $\left(\sim 80^{\circ} \mathrm{C}\right.$, without detergent), to remove traces of detergent or residual beads. Particular attention must be paid to any trapped areas or crevices.

- Blow dry with nitrogen.

- Immerse completely in an ultrasonically-agitated bath of clean hot $\left(\sim 80^{\circ} \mathrm{C}\right)$ stabilized solvent for at least 15 minutes. Some solvents that could be used are:

○ 1:100 by volume Liquinox $(\mathrm{PH}=8.5)$

○ $10-20 \mathrm{~mL}$ per litre Citranox $®(\mathrm{PH}=2.5)$.

- Wash with hot water $\left(\sim 80^{\circ} \mathrm{C}\right)$, using clean de-ionized water (minimum resistance $=1,000,000 \mathrm{Ohm} / \mathrm{cm})$. Detergent must not be used at this stage.

- Blow dry with nitrogen.

- If assembly cannot be done immediately wrap in UHV grade aluminium foil.

To perform the ultrasonic cleaning a 40-cm-long titanium ultrasonic probe compatible with a $700-\mathrm{W}$ sonicator has been custom designed. This probe is used to clean the surface of large components by exposing them to the micro bubbles crated by the $-40 \mathrm{kHz}$ vibration of the probe. The bubbles blast the surface, getting rid of contaminants. Also a large, 30" x 30" x 40" aluminium sink has been fabricated so the largest components can be fully submerged into the detergent solutions and sonicated. For smaller components a Branson ultrasonic cleaner is used. To facilitate assembly and future additions/modifications a crane has been designed and built. The crane, shown in Figure A.7, has up to $2000 \mathrm{lb}$ capacity and up to 10 feet longitudinal reach and 8 feet maximum hoisting height. 


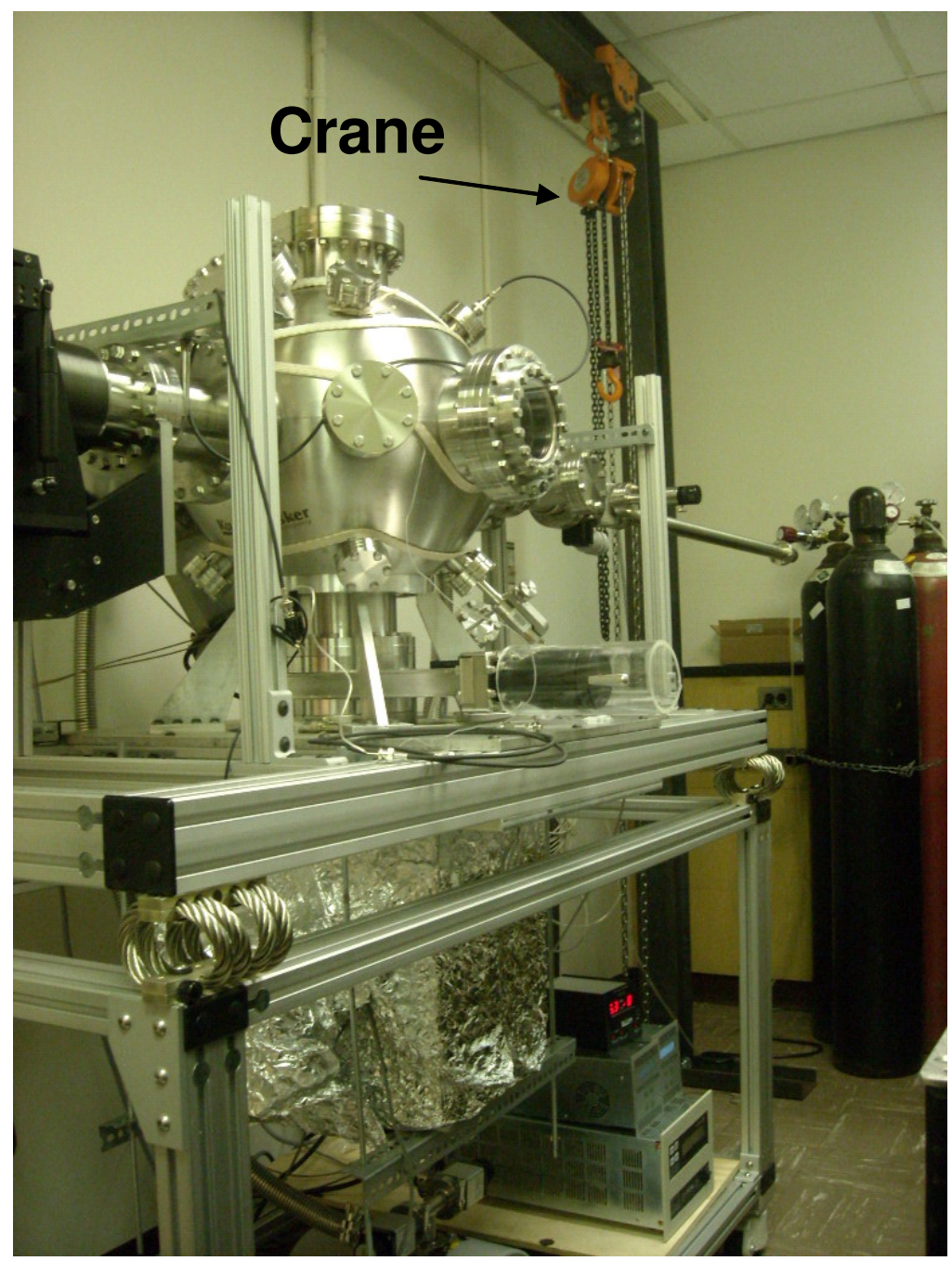

Figure A.7: Isometric view of the system. Electronic controllers, assembly crane and nitrogen tanks are visible.

\section{A.3 Future Work}

The main chamber of this UHV system has several additional ports and can be further expanded. Further planned expansions include the addition of an activation chamber and transfer unit. This chamber will allow for in-situ treatment of devices, thus avoiding the contamination produced by exposure to the ambient. Additionally, the system is currently operated manually but the operation can be automated by a centralized computer interface for all the controllers. 


\section{B Supplementary Material for Chapter 2}

\section{B.1 Oxide Charge Calculation}

Oxide charging can be calculated as a function of the primary beam energy and current. The following equation can be used to calculate the charge left in the substrate (based on the model presented in [1]):

$$
I_{B}=\delta I_{B}+\eta I_{B}+I_{S C}
$$

, where $I_{B}$ is the beam current and $\delta$ and $\eta$ are the oxide secondary electron yield and backscattered electron yield coefficients, respectively, at the primary beam energy in use. $I_{S C}$ is the leakage current due to the finite resistance of the dielectric, surface contamination and electron beam induced currents that occur when the primary beam electrons penetrate beyond the dielectric layer. At low beam energies the latter component is zero. As the substrate is bombarded with electrons some of them will be reflected due to elastic and inelastic collisions. Elastic collisions will generate backscattered electrons with energies ranging from $50 \mathrm{eV}$ to the primary beam energy. Inelastic collisions occur between the primary beam electrons or backscattered electrons and the nuclei of the dielectric, and generate electrons with less than 50 $\mathrm{eV}$ (secondaries). The majority of reflected electrons will be of this type. The reflected electron yield, which is the sum of secondary and backscattered electron yields, is a function of the primary beam energy and the tilt of the sample. This yield will be equal to unity at two beam energies. For a beam normal to the sample these energies are less than $400 \mathrm{eV}$ and approximately $3 \mathrm{keV}$ for silicon dioxide (we call them $\mathrm{E}_{1}$ and $\mathrm{E}_{2}$, respectively) $[1,2,3]$. For energies between $E_{1}$ and $E_{2}$ the reflected electron yield is larger than unity and everywhere else it is less than unity.

At the primary beam energies used in our experiments ( $3 \mathrm{keV}$ or more) the yield is less than unity and some extra electrons will be left in the substrate, charging it negatively. This charge, however, will in turn raise the surface energy, $E_{\text {surface }}$, reducing the effective landing energy $E^{\prime}$ of 
the primary beam electrons. The increased surface potential will also cause an increase in the leakage current through the oxide. The following equation can be used to calculate $E_{\text {surface }}$ and the landing energy $E^{\prime}[1]$ :

$$
E_{\text {Surface }}=\frac{I_{B} R f_{0}\left(E_{0}-E_{2}\right)}{\left(E_{0}-E_{2}\right)+I_{B} R f_{0}}
$$

The landing energy is $E^{\prime}=E_{0}-E_{\text {Surface }}$ and $E_{o}$ is the primary beam energy. The charging coefficient is $f_{0}=1-(\delta+\eta)_{E^{\prime}}$ and $R$ is the bulk resistance, which is a function of the volume of all the current paths in the $\mathrm{SiO}_{2}$. From the landing energy $E$ ' the substrate charging can be calculated using the secondary and backscatter yield coefficients at this energy and using equation (1). We implemented the above equations to calculate the substrate charging with a Matlab script using the $\mathrm{SiO}_{2}$ backscattered and secondary electron yield coefficients published by Joy [4]. The values of yield coefficients not published in Joy's database were linearly interpolated. 


\section{B.2 Bibliography}

[1] H. Seiler, Journal of Applied Physics 54, R1, (1983).

[2] D. C. Joy and C. S. Joy, Micron 27, 247, (1996).

[3] L. Reimer, (1993), Image formation in Low Voltage Electron Microscopy, Bellingham, WA: SPIE.

[4] D. C. Joy, A Database of Electron-Solid Interactions, 2008, http://web.utk.edu/ srcutk/htm/interact.htm. 